\title{
Conservação pós-colheita de frutos de Passiflora setacea DC. submetidos a diferentes sanitizantes e temperaturas de armazenamento
}

\section{Post-harvest conservation of Passiflora setacea DC. fruits submitted to different sanitizers and storage temperatures}

\author{
Maria Madalena Rinaldi, Ana Maria Costa', Fabio Gelape Faleiro', Nilton Tadeu Vilela Junqueira ${ }^{1}$ \\ Embrapa Cerrados (CPAC), Planaltina/DF - Brasil

\section{${ }^{*}$ Corresponding Author} \\ Maria Madalena Rinaldi, Embrapa Cerrados (CPAC), BR 020, km 18, Caixa Postal: 08223, CEP: 73310-970, Planaltina/DF - Brasil, e-mail: madalena. \\ rinaldi@embrapa.br
}

Cite as: Post-harvest conservation of Passiflora setacea DC. fruits submitted to different sanitizers and storage temperatures. Braz. J. Food Technol., v. 20, e2016046, 2017.

Received: Apr. 15, 2016; Accepted: May 03, 2017

\section{Resumo}

A Passiflora setacea DC. é uma espécie silvestre de maracujazeiro que ocorre nos biomas Cerrado e Caatinga, e em áreas de transição, como o Semiárido norte-mineiro do Brasil. A alta perecibilidade dos frutos de $P$. setacea reduz sua vida útil, limitando sua comercialização. Objetivou-se determinar a melhor temperatura e o melhor sanitizante para a conservação pós-colheita de frutos de $P$. setacea. Os frutos foram submetidos a dois sanitizantes químicos e armazenados por 21 dias, sob condição ambiente $\left(21,3{ }^{\circ} \mathrm{C}\right.$ e $77,8 \%$ de umidade relativa) e nas temperaturas de $6{ }^{\circ} \mathrm{C}, 10{ }^{\circ} \mathrm{C}, 15{ }^{\circ} \mathrm{C}$ e $20{ }^{\circ} \mathrm{C}$ e $90 \%$ de umidade relativa. No início do armazenamento e aos 4, 7, 14 e 21 dias, realizaram-se análises de perda de massa fresca, cor, acidez titulável, sólidos solúveis, ratio e pH dos frutos. A temperatura de $10^{\circ} \mathrm{C}$ e $90 \%$ de umidade relativa é a condição mais recomendada para o armazenamento dos frutos. Os sanitizantes não aumentaram a vida útil dos frutos, não sendo recomendados para utilização nas concentrações estudadas, uma vez que os mesmos não auxiliaram na redução da perda de massa fresca e na conservação dos mesmos. De acordo com as condições experimentais do presente trabalho, a vida útil pós-colheita dos frutos foi inferior a quatro dias de armazenamento, mesmo sob refrigeração; observou-se que, assim como o maracujazeiro azedo comercial, frutos de $P$. setacea perdem massa fresca e apresentam o enrugamento da casca após quatro dias de armazenamento. No entanto, de acordo com os valores de acidez titulável, sólidos solúveis e pH, essas características não inviabilizam o consumo da polpa após sete dias de armazenamento.

Palavras-chave: Físico-química; Maracujá; Refrigeração; Sanitização; Vida útil.

\section{Abstract}

Passiflora setacea DC. is a wild passion fruit species that occurs in the savanna (Cerrado) and scrubland (Caatinga) biomes of Brazil and transitional areas such as the semi-arid northern area of Minas Gerais State. The high perishability of the $P$. setacea fruits reduces their shelf life and limits their marketing. The objective was to determine the best temperature and best sanitizing agent for the post-harvest conservation of $P$. setacea fruits. The fruits were subjected to two chemical sanitizers and stored for 21 days under environmental conditions at temperatures of $6{ }^{\circ} \mathrm{C}, 10^{\circ} \mathrm{C}, 15{ }^{\circ} \mathrm{C}$ and $20^{\circ} \mathrm{C}$ at $90 \%$ of relative humidity. At zero storage time and after 4, 7, 14 and 21 days, the fruits were analysed for loss of weight, colour, titratable acidity, soluble solids, ratio and $\mathrm{pH}$. The most recommended conditions for storage of the fruits were a temperature of $10^{\circ} \mathrm{C}$ and relative humidity of $90 \%$. The sanitizers did not increase the shelf life of the fruits and are not recommended for use at the concentrations studied since they did not contribute to a reduction in the loss of fresh weight or conservation of the fruits. According to the experimental conditions of the present study, the post-harvest shelf life of the fruit was less than four days, even under refrigerated storage. Similar to the commercial acid passion fruit, the $P$. setacea fruits lose fresh weight and the peel wrinkles after four days of storage. However according to the values obtained for $\mathrm{pH}$, titratable acidity and soluble solids, these characteristics do not preclude the use of the pulp after seven days of storage.

Keywords: Physicochemical; Passion fruit; Refrigeration; Sanitization; Shelf life. 
Rinaldi, M. M. et al.

\section{Introdução}

O Brasil é o principal produtor mundial de maracujá, com produção, em 2014, de 823 mil toneladas, principalmente na região Nordeste, sendo o Estado da Bahia o maior produtor. A Região Centro-Oeste situa-se atualmente em quarto lugar na produção nacional de maracujá (INSTITUTO FNP, 2016). No Brasil, ações de pesquisa e desenvolvimento têm evidenciado o potencial agronômico e comercial de outras espécies silvestres de Passiflora, além da espécie Passiflora edulis Sims, como a Passiflora setacea DC. (CERQUEIRA-SILVA et al., 2014; FALEIRO et al., 2014).

Em frutos de maracujá destinados ao mercado in natura, o critério mais utilizado para avaliar sua qualidade é a aparência externa, sendo que um dos problemas identificados pela cadeia produtiva para a sua comercialização é a perda de massa e o consequente murchamento, o que confere aspecto enrugado ao fruto. Além do murchamento, também apresentam grande susceptibilidade à podridão e à fermentação da polpa, o que resulta em curta vida útil (TAVARES et al., 2003; DURIGAN, 1998)

Após a colheita, de maneira geral, os frutos de maracujá de diferentes espécies apresentam vida útil reduzida de apenas três a sete dias, em condições de temperatura ambiente. Para frutos de Passiflora setacea, ainda não é conhecido o período de conservação pós-colheita dos mesmos, de forma que estudos de pós-colheita utilizando-se diferentes sanitizantes e temperaturas de armazenamento foram realizados com o objetivo de definir a sua vida útil. Os sanitizantes, aliados à temperatura de armazenamento adequada, podem auxiliar no aumento da vida útil, reduzindo a contaminação microbiana e as doenças pós-colheita, e na menor perda de massa fresca, resultando em maior tempo dos frutos em condições de consumo.

A espécie Passiflora setacea é nativa dos Cerrados brasileiros e apresenta grande potencial para consumo in natura, em virtude do aroma agradável e adocicado dos seus frutos (ATAÍDE et al., 2012). Há 20 anos, foi iniciado um programa de melhoramento genético de $P$. setacea, realizado pela Embrapa e parceiros, o qual culminou no lançamento da cultivar BRS Pérola do Cerrado (BRS PC), em 2013 (EMBRAPA, 2015).

A cultivar BRS Pérola do Cerrado tem conquistado os produtores e consumidores brasileiros, e a cadeia produtiva está sendo estabelecida e fortalecida a cada ano (EMBRAPA, 2015). Assim, é de grande importância o aprofundamento das informações sobre a qualidade e a conservação pós-colheita de frutos de Passiflora setacea produzidos e comercializados no Brasil. Nesse sentido, objetivou-se determinar a vida útil de frutos de Passiflora setacea submetidos a diferentes sanitizantes e temperaturas de armazenamento.

\section{Material e métodos}

Frutos da espécie Passiflora setacea DC. cv. BRS Pérola do Cerrado, oriundos do campo experimental da Embrapa Cerrados, foram colhidos no ponto de maturação comercial, em que os frutos apresentam coloração verde-clara com listras verde-escuras em sentido longitudinal e a polpa, cor amarelo-clara ou creme; as sementes são obovadas levemente reticuladas, com cerca de $0,5 \mathrm{~cm}$ de diâmetro por $0,3 \mathrm{~cm}$ de comprimento. Os frutos foram lavados em água corrente e imersos (10 minutos) em solução sanificante de hipoclorito de sódio (hipoclorito inorgânico - HI) com $150 \mathrm{mg} \mathrm{L}^{-1}$ de cloro ativo. Amostras de frutos também foram imersos por 10 minutos em sanitizante (hipoclorito orgânico - HO) para hortifrutícolas, com nome comercial SanitVeg ${ }^{\circledR}$ da empresa Perol Comércio e Indústria LTDA. de Ribeirão Preto - SP, em concentração conforme recomendações do fabricante. Para o enxague, o tempo de imersão foi de 5 minutos em solução de $5 \mathrm{mg} \mathrm{L}^{-1}$, de acordo com o sanitizante utilizado (hipoclorito orgânico e hipoclorito inorgânico), com posterior drenagem dos frutos (5 minutos) e secagem em papel toalha. O tratamento testemunha consistiu apenas na lavagem dos frutos em água corrente, com posterior secagem em papel toalha.

Os frutos foram armazenados sob refrigeração em câmara fria com umidade relativa de $90 \%$ nas temperaturas de $6{ }^{\circ} \mathrm{C}, 10^{\circ} \mathrm{C}, 15^{\circ} \mathrm{C}$ e $20^{\circ} \mathrm{C}$. Para a simulação de condição ambiente, os frutos também foram mantidos em câmara fria na temperatura de $21,3^{\circ} \mathrm{C}$ e de $77,8 \%$ UR. O período de armazenamento para todos os tratamentos foi de 21 dias.

Somente no início do experimento, os frutos foram pesados, sendo também medidos o comprimento (diâmetro do fruto do ponto de inserção do pecíolo no fruto até o ápice do fruto) e a largura (maior medida do diâmetro do fruto, perpendicular ao seu comprimento). Calculou-se também o índice de formato do fruto, pela relação comprimento e largura. Foram obtidos o peso da polpa mais as sementes, o peso da polpa, o peso das sementes, o peso das cascas e o volume de polpa. No início do armazenamento e aos 4, 7, 14 e 21 dias, o produto foi submetido à análise de perda de massa fresca dos frutos pela diferença de peso entre a massa inicial e a massa no momento da avaliação; a cor da casca dos frutos $\left(L^{*}, a^{*}, b^{*}\right)$ foi determinada em espectrofotômetro MiniScan ${ }^{\circledR}$ EZ marca HunterLab, sendo realizadas cinco leituras por fruto de Passiflora setacea. $O$ valor de $L^{*}$ define a luminosidade $\left(L^{*}=0\right.$ preto $e L^{*}=100$ branco) e $a^{*}$ e b* são responsáveis pela cromaticidade ( $+a^{*}$ vermelho e $-a^{*}$ verde), $b^{*}\left(+b^{*}\right.$ amarelo e $-b^{*}$ azul). Por meio do módulo $L^{*}$, $a^{*} e b^{*}$, calculou-se o incremento no escurecimento $\left\{\left[\left(L^{*}-L^{*} 0\right)^{2}+\left(a^{*}-a^{*} 0\right)^{2}+\left(b^{*}-b^{*} 0\right)^{2}\right]^{1 / 2}\right\}$, chroma (saturação 
Rinaldi, M. M. et al.

ou intensidade da cor; 0 - cor impura e 60 - cor pura) e o ângulo hue (ângulo da cor; $0^{\circ}$ vermelho; $90^{\circ}$ amarelo; $180^{\circ}$ verde; $270^{\circ}$ azul e $360^{\circ}$ negro), por meio das fórmulas: chroma $\left[\left(a^{2}+b^{2}\right)^{1 / 2}\right]$ e ângulo hue [arco tangente $\left.(b / a)\right]$ para $a^{*}$ positivo, e [arco tangente $\left.\left(b^{*} / a^{*}\right)(-1)+90\right]$ para $a^{*}$ negativo, conforme recomendado por Hunterlab (2008). Acidez titulável, sólidos solúveis, ratio e pH foram medidos de acordo com Carvalho et al. (1990).

Utilizou-se o delineamento experimental inteiramente casualizado, em esquema fatorial $3 \times 5$, sendo três tipos de sanitizantes $\left(\mathrm{H}_{2} \mathrm{O}, \mathrm{HI}\right.$ e $\left.\mathrm{HO}\right)$ e cinco temperaturas (AMB, $6{ }^{\circ} \mathrm{C}, 10^{\circ} \mathrm{C}, 15^{\circ} \mathrm{C}$ e $20^{\circ} \mathrm{C}$ ) de armazenamento, para cada dia de análise. Compararam-se também os três tipos de sanitizantes $\left(\mathrm{H}_{2} \mathrm{O}, \mathrm{Hl}\right.$ e HO$)$ em relação aos cinco dias de análise (0, 4, 7, 14 e 21 dias), para cada temperatura. Para cada tratamento, utilizaram-se três repetições, sendo que cada uma consistiu em cinco frutos de Passiflora setacea. Para as análises de cor, foram utilizados os cinco frutos por tratamento, sendo realizadas cinco leituras por fruto em cada dia de análise, sempre no mesmo lote de frutos, do início ao final do armazenamento. Os dados foram submetidos à análise de variância utilizando-se o teste $\mathrm{F}$ e as médias foram comparadas por meio do teste Tukey a $5 \%$ de probabilidade de erro. Todas as análises estatísticas foram realizadas utilizando-se o software ASSISTAT (SILVA, 2015).

\section{Resultados e discussão}

\subsection{Caracterização inicial}

O peso médio dos frutos foi de $51,30 \mathrm{~g}$, sendo este superior aos valores obtidos por Ataíde et al. (2012), em frutos de Passiflora setacea produzidos em Jaboticabal - SP. O comprimento foi de $5,25 \mathrm{~cm}$ e a largura de $4,54 \mathrm{~cm}$. O índice de formato do fruto foi de 0,86 , correspondendo a frutos redondo-ovalados, de acordo com a classificação realizada por Fortaleza et al. (2005). As demais características físicas dos frutos foram assim determinadas: massa da polpa mais sementes: 20,98 g; massa da polpa: $12,90 \mathrm{~g}$; porcentagem de polpa: $34,08 \%$, superando o valor de $33 \%$, que é o limite mínimo utilizado como padrão no processamento industrial de Passiflora edulis. Ataíde et al. (2012) obtiveram rendimento de polpa similar (34,52\%). Outras variáveis determinadas: massa das sementes: 8,08 g; porcentagem de sementes: 20,41\%; peso das cascas: $27,80 \mathrm{~g}$; porcentagem de cascas; $89,10 \%$, e volume de polpa: $12,91 \mathrm{~mL}$ por fruto.

\subsection{Análises físico-químicas}

A menor perda de massa fresca ocorreu nos frutos armazenados na temperatura de $6{ }^{\circ} \mathrm{C}$ (Tabela 1). Em produtos vegetais, quanto menor a temperatura de armazenamento, menor é a taxa respiratória, resultando em menor transpiração e perda de massa fresca; justifica-se, assim, a menor perda de massa nos frutos armazenados a $6{ }^{\circ} \mathrm{C}$. Os produtos perecíveis, mesmo quando mantidos em condições ideais de temperatura e umidade, sofrem alguma perda de massa durante 0 armazenamento, devido ao efeito combinado da respiração e da transpiração (CHITARRA; CHITARRA, 2005). Ocorreu perda significativa de massa fresca entre os tratamentos (Tabela 1 ), sendo maior nos frutos submetidos à temperatura ambiente, em que a umidade relativa foi significativamente menor durante todo o experimento.

O controle da perda de massa e do enrugamento do maracujá-amarelo tem fundamental importância, pois sua comercialização é realizada por peso e aparência (ROTILI et al., 2013). Geralmente, em temperaturas mais altas, a perda de massa fresca dos frutos de maracujás é maior. Neste experimento, aos 4, 7 e 14 dias de armazenamento, em alguns tratamentos, os frutos armazenados na temperatura de $15{ }^{\circ} \mathrm{C}$ apresentaram maior porcentagem de perda de massa fresca, quando comparados aos mantidos na temperatura de $20^{\circ} \mathrm{C} \mathrm{com}$ a mesma porcentagem de umidade relativa no ambiente de armazenamento. Provavelmente, isso ocorreu devido a características intrínsecas dos frutos submetidos aos diferentes tratamentos. Em frutos de maracujá amarelo, os sintomas de senescência, como o enrugamento da casca, iniciam entre três e sete dias após sua abscisão, havendo a necessidade de serem comercializados imediatamente após a colheita (VENÂNCIO et al., 2013).

Frutos de maracujazeiro-amarelo são considerados murchos a partir de uma perda de massa fresca de $8 \%$ do seu peso inicial, por prejudicar a aparência, depreciando o seu valor comercial (FAEP, 2015). No presente trabalho, apenas os frutos somente lavados com água corrente e mantidos na temperatura de $6{ }^{\circ} \mathrm{C}$ não atingiram esta porcentagem até os quatro dias de armazenamento. Os demais tratamentos, aos quatro dias, apresentaram valores significativamente superiores de perda de massa fresca. De maneira geral, os frutos submetidos aos sanitizantes não apresentaram redução na perda de massa fresca durante o armazenamento, provavelmente devido à retirada da película protetora natural na superfície dos frutos pelos produtos utilizados.

Não ocorreu variação significativa nos valores de luminosidade da casca dos frutos durante o armazenamento (tabela de valores não apresentada). Os valores variaram entre 39,18 e 55,74, com valor médio de 48,88. O maior valor foi apresentado pelo produto lavado apenas com $\mathrm{H}_{2} \mathrm{O}$ e armazenado a $10{ }^{\circ} \mathrm{C}$, aos quatro dias de armazenamento. $\mathrm{O}$ menor valor foi obtido no produto também lavado com $\mathrm{H}_{2} \mathrm{O}$ e armazenado a $15{ }^{\circ} \mathrm{C}$, aos 14 dias de armazenamento. Na temperatura de $6{ }^{\circ} \mathrm{C}$ e $90 \%$ de umidade relativa, ocorreu dano pelo frio na parte externa dos frutos, denominado chilling injury, sendo mais 
Conservação pós-colheita de frutos de Passiflora setacea DC. submetidos a diferentes sanitizantes e temperaturas de armazenamento

Rinaldi, M. M. et al.

Tabela 1. Valores médios de perda de massa fresca em frutos de Passiflora setacea cv. BRS Pérola do Cerrado, submetidos a diferentes tratamentos.

\begin{tabular}{|c|c|c|c|c|c|c|c|}
\hline \multirow{5}{*}{ TEMP } & \multicolumn{7}{|c|}{ Perda de massa fresca (\%) } \\
\hline & \multicolumn{3}{|c|}{ DA } & \multirow{4}{*}{ DA } & \multicolumn{3}{|c|}{ TEMP } \\
\hline & \multicolumn{3}{|c|}{ Sanitizante } & & \multicolumn{3}{|c|}{ Sanitizante } \\
\hline & $\mathrm{H}_{2} \mathrm{O}$ & $\mathrm{HI}$ & $\mathrm{HO}$ & & $\mathrm{H}_{2} \mathrm{O}$ & HI & $\mathrm{HO}$ \\
\hline & \multicolumn{3}{|c|}{ Dia 0} & & \multicolumn{3}{|c|}{ AMB } \\
\hline AMB & $0,00 \mathrm{aA}$ & $0,00 \mathrm{aA}$ & $0,00 \mathrm{aA}$ & 0 & $0,00 \mathrm{cA}$ & $0,00 \mathrm{cA}$ & $0,00 \mathrm{cA}$ \\
\hline $6^{\circ} \mathrm{C}$ & $0,00 \mathrm{aA}$ & $0,00 \mathrm{aA}$ & $0,00 \mathrm{aA}$ & 4 & $20,42 \mathrm{bA}$ & $21,68 \mathrm{bA}$ & $22,36 \mathrm{bA}$ \\
\hline $10^{\circ} \mathrm{C}$ & $0,00 \mathrm{aA}$ & $0,00 \mathrm{aA}$ & $0,00 \mathrm{aA}$ & 7 & 31,50 aA & $33,24 \mathrm{aA}$ & 34,89 aA \\
\hline $15^{\circ} \mathrm{C}$ & $0,00 \mathrm{aA}$ & 0,00 aA & $0,00 \mathrm{aA}$ & 14 & NA & NA & NA \\
\hline $20{ }^{\circ} \mathrm{C}$ & $0,00 \mathrm{aA}$ & $0,00 \mathrm{aA}$ & $0,00 \mathrm{aA}$ & 21 & NA & NA & NA \\
\hline \multicolumn{5}{|c|}{ Dia 4} & \multicolumn{3}{|c|}{$6^{\circ} \mathrm{C}$} \\
\hline AMB & $20,42 \mathrm{aA}$ & $21,68 \mathrm{aA}$ & $22,36 \mathrm{aA}$ & 0 & 0,00 eA & 0,00 eA & 0,00 eA \\
\hline $6^{\circ} \mathrm{C}$ & $6,16 \mathrm{~dB}$ & $9,52 \mathrm{cA}$ & $10,09 \mathrm{bcA}$ & 4 & $6,16 \mathrm{~dB}$ & $9,52 \mathrm{dAB}$ & $10,09 \mathrm{dA}$ \\
\hline $10^{\circ} \mathrm{C}$ & $8,54 \mathrm{cdA}$ & $9,05 \mathrm{cA}$ & $9,11 \mathrm{cA}$ & 7 & $10,40 \mathrm{cB}$ & $15,86 \mathrm{cA}$ & $16,37 \mathrm{cA}$ \\
\hline $15^{\circ} \mathrm{C}$ & $11,88 \mathrm{bA}$ & $11,48 \mathrm{bcA}$ & $12,54 \mathrm{bA}$ & 14 & $20,14 \mathrm{bB}$ & 27,02 bA & $27,57 \mathrm{bA}$ \\
\hline $20{ }^{\circ} \mathrm{C}$ & $9,23 \mathrm{cB}$ & $12,15 \mathrm{bA}$ & 10,13 bcAB & 21 & $30,64 \mathrm{aB}$ & 36,10 aA & 37,15 aA \\
\hline \multicolumn{5}{|c|}{ Dia 7} & \multicolumn{3}{|c|}{$10^{\circ} \mathrm{C}$} \\
\hline $\mathrm{AMB}$ & $31,50 \mathrm{aB}$ & 33,24 aAB & $34,87 \mathrm{aA}$ & 0 & $0,00 \mathrm{eA}$ & 0,00 eA & 0,00 eA \\
\hline $6^{\circ} \mathrm{C}$ & $10,40 \mathrm{~dB}$ & 15,86 bA & $16,37 \mathrm{bA}$ & 4 & $8,54 \mathrm{dA}$ & $9,05 \mathrm{dA}$ & $9,11 \mathrm{dA}$ \\
\hline $10^{\circ} \mathrm{C}$ & $14,17 \mathrm{bcA}$ & $16,10 \mathrm{bA}$ & $15,28 \mathrm{bA}$ & 7 & $14,17 \mathrm{cA}$ & $16,10 \mathrm{cA}$ & $15,28 \mathrm{cA}$ \\
\hline $15^{\circ} \mathrm{C}$ & $16,62 \mathrm{bA}$ & $15,57 \mathrm{bA}$ & 16,23 bA & 14 & $25,17 \mathrm{bB}$ & 29,12 bA & $28,00 \mathrm{bA}$ \\
\hline $20{ }^{\circ} \mathrm{C}$ & $12,81 \mathrm{cdB}$ & $16,10 \mathrm{bA}$ & $13,75 \mathrm{bAB}$ & 21 & $35,75 \mathrm{aB}$ & $41,33 \mathrm{aA}$ & 39,97 aA \\
\hline \multicolumn{5}{|c|}{ Dia 14} & \multicolumn{3}{|c|}{$15^{\circ} \mathrm{C}$} \\
\hline AMB & NA & NA & NA & 0 & $0,00 \mathrm{dA}$ & $0,00 \mathrm{dA}$ & $0,00 \mathrm{dA}$ \\
\hline $6{ }^{\circ} \mathrm{C}$ & $20,14 \mathrm{cA}$ & $27,02 \mathrm{cbA}$ & $27,57 \mathrm{aA}$ & 4 & $11,88 \mathrm{cA}$ & $11,48 \mathrm{cA}$ & $12,54 \mathrm{cA}$ \\
\hline $10^{\circ} \mathrm{C}$ & $25,17 \mathrm{bA}$ & $29,12 \mathrm{abA}$ & $28,00 \mathrm{aA}$ & 7 & $16,62 \mathrm{bA}$ & $15,57 \mathrm{bA}$ & $16,23 \mathrm{bA}$ \\
\hline $15^{\circ} \mathrm{C}$ & 29,68 aA & $28,31 \mathrm{bA}$ & 28,29 aA & 14 & 29,68 aA & $28,31 \mathrm{aA}$ & 28,29 aA \\
\hline $20^{\circ} \mathrm{C}$ & 28,33 aA & 32,88 aA & $29,57 \mathrm{aA}$ & 21 & NA & NA & NA \\
\hline \multicolumn{5}{|c|}{ Dia 21} & \multicolumn{3}{|c|}{$20^{\circ} \mathrm{C}$} \\
\hline AMB & NA & NA & NA & 0 & $0,00 \mathrm{dA}$ & $0,00 \mathrm{dA}$ & $0,00 \mathrm{dA}$ \\
\hline $6^{\circ} \mathrm{C}$ & 30,64 bA & $36,10 \mathrm{bA}$ & $37,15 \mathrm{aA}$ & 4 & $9,23 \mathrm{cA}$ & $12,15 \mathrm{cA}$ & $10,13 \mathrm{cA}$ \\
\hline $10^{\circ} \mathrm{C}$ & 35,75 aA & $41,33 \mathrm{aA}$ & $39,97 \mathrm{aA}$ & 7 & $12,81 \mathrm{bA}$ & $16,10 \mathrm{bA}$ & $13,75 \mathrm{bA}$ \\
\hline $15^{\circ} \mathrm{C}$ & NA & NA & NA & 14 & 28,33 aA & 32,88 aA & 29,57 aA \\
\hline $20^{\circ} \mathrm{C}$ & NA & NA & NA & 21 & NA & NA & NA \\
\hline
\end{tabular}

TEMP: temperatura; DA: dia de análise; AMB: ambiente; HI: hipoclorito inorgânico; HO: hipoclorito orgânico; NA: Não Analisado. Letras minúsculas iguais, na mesma coluna, não diferem significativamente no nível de $5 \%$ no teste de Tukey. Letras maiúsculas iguais, na mesma linha, não diferem significativamente no nível de 5\% no teste de Tukey.

recomendada a temperatura de $10^{\circ} \mathrm{C}$ e $90 \%$ de umidade relativa, para o armazenamento dos frutos.

A medida do incremento no escurecimento tem o objetivo de verificar o escurecimento do produto durante 0 armazenamento. De maneira geral, para produtos vegetais, considera-se que, quando os valores de $\mathrm{E}>10$, o grau de escurecimento é elevado. Os valores de incremento no escurecimento nos frutos variaram entre zero e 20,31 (Tabela 2). O maior incremento no escurecimento ocorreu nos primeiros quatro dias de armazenamento em todos os tratamentos, com maior valor no produto submetido à sanitização com hipoclorito orgânico e mantido na temperatura de $6{ }^{\circ} \mathrm{C}$. Provavelmente, o chilling injury influenciou no escurecimento dos frutos. De maneira geral, ocorreu incremento no escurecimento significativo em todos os tratamentos, durante o armazenamento. Dessa forma, não é possível afirmar que a utilização da refrigeração aliada a alguns sanitizantes em frutos de Passiflora setacea irá auxiliar na manutenção da cor dos frutos. 
Conservação pós-colheita de frutos de Passiflora setacea DC. submetidos a diferentes sanitizantes e temperaturas de armazenamento

Rinaldi, M. M. et al.

Tabela 2. Valores médios de incremento no escurecimento em frutos de Passiflora setacea cv. BRS Pérola do Cerrado, submetidos a diferentes tratamentos.

\begin{tabular}{|c|c|c|c|c|c|c|c|}
\hline \multirow{5}{*}{ TEMP } & \multicolumn{7}{|c|}{ Incremento no escurecimento } \\
\hline & \multicolumn{3}{|c|}{ DA } & \multirow{4}{*}{ DA } & \multicolumn{3}{|c|}{ TEMP } \\
\hline & \multicolumn{3}{|c|}{ Sanitizante } & & \multicolumn{3}{|c|}{ Sanitizante } \\
\hline & $\mathrm{H}_{2} \mathrm{O}$ & $\mathrm{HI}$ & $\mathrm{HO}$ & & $\mathrm{H}_{2} \mathrm{O}$ & $\mathrm{HI}$ & HO \\
\hline & \multicolumn{3}{|c|}{ Dia 0} & & \multicolumn{3}{|c|}{ AMB } \\
\hline AMB & 0,00 aA & $0,00 \mathrm{aA}$ & $0,00 \mathrm{aA}$ & 0 & $0,00 \mathrm{bA}$ & $0,00 \mathrm{cA}$ & $0,00 \mathrm{bA}$ \\
\hline $6^{\circ} \mathrm{C}$ & $0,00 \mathrm{aA}$ & $0,00 \mathrm{aA}$ & $0,00 \mathrm{aA}$ & 4 & $15,33 \mathrm{aB}$ & $19,95 \mathrm{aA}$ & $15,12 \mathrm{aB}$ \\
\hline $10^{\circ} \mathrm{C}$ & 0,00 aA & 0,00 aA & $0,00 \mathrm{aA}$ & 7 & $15,72 \mathrm{aA}$ & $16,98 \mathrm{bA}$ & $17,99 \mathrm{aA}$ \\
\hline $15^{\circ} \mathrm{C}$ & $0,00 \mathrm{aA}$ & $0,00 \mathrm{aA}$ & $0,00 \mathrm{aA}$ & 14 & NA & NA & NA \\
\hline $20{ }^{\circ} \mathrm{C}$ & $0,00 \mathrm{aA}$ & $0,00 \mathrm{aA}$ & $0,00 \mathrm{aA}$ & 21 & NA & NA & NA \\
\hline \multicolumn{5}{|c|}{ Dia 4} & \multicolumn{3}{|c|}{$6{ }^{\circ} \mathrm{C}$} \\
\hline AMB & $15,33 \mathrm{abA}$ & 19,95 aA & $15,12 \mathrm{bA}$ & 0 & $0,00 \mathrm{cA}$ & $0,00 \mathrm{bA}$ & $0,00 \mathrm{bA}$ \\
\hline $6^{\circ} \mathrm{C}$ & $16,12 \mathrm{aA}$ & $18,64 \mathrm{aA}$ & $20,31 \mathrm{aA}$ & 4 & $16,12 \mathrm{abA}$ & $18,64 \mathrm{aA}$ & $20,31 \mathrm{aA}$ \\
\hline $10^{\circ} \mathrm{C}$ & $18,00 \mathrm{aA}$ & $17,51 \mathrm{aA}$ & $19,98 \mathrm{aA}$ & 7 & $18,72 \mathrm{aA}$ & $17,52 \mathrm{aA}$ & $20,06 \mathrm{aA}$ \\
\hline $15^{\circ} \mathrm{C}$ & $13,66 \mathrm{bA}$ & $13,85 \mathrm{bA}$ & $13,67 \mathrm{cA}$ & 14 & $16,83 \mathrm{abA}$ & $17,99 \mathrm{aA}$ & $19,99 \mathrm{aA}$ \\
\hline $20^{\circ} \mathrm{C}$ & $12,73 \mathrm{cbA}$ & $13,51 \mathrm{bA}$ & $13,98 \mathrm{bcA}$ & 21 & $15,10 \mathrm{bA}$ & $18,70 \mathrm{aA}$ & $18,83 \mathrm{aA}$ \\
\hline \multicolumn{5}{|c|}{ Dia 7} & \multicolumn{3}{|c|}{$10^{\circ} \mathrm{C}$} \\
\hline AMB & $15,72 \mathrm{bA}$ & $16,98 \mathrm{abA}$ & 17,99 aA & 0 & $0,00 \mathrm{bA}$ & $0,00 \mathrm{cA}$ & $0,00 \mathrm{cA}$ \\
\hline $6^{\circ} \mathrm{C}$ & $18,72 \mathrm{aA}$ & $17,52 \mathrm{aA}$ & $20,06 \mathrm{aA}$ & 4 & $18,00 \mathrm{aA}$ & $17,51 \mathrm{abA}$ & $19,98 \mathrm{aA}$ \\
\hline $10^{\circ} \mathrm{C}$ & $18,09 \mathrm{aA}$ & $15,63 \mathrm{abA}$ & $16,54 \mathrm{bA}$ & 7 & $18,09 \mathrm{aA}$ & $15,63 \mathrm{bA}$ & $16,54 \mathrm{bA}$ \\
\hline $15^{\circ} \mathrm{C}$ & $12,25 \mathrm{cA}$ & $15,03 \mathrm{bA}$ & $13,98 \mathrm{cA}$ & 14 & 17,76 aA & $19,63 \mathrm{aA}$ & 20,06 aA \\
\hline $20{ }^{\circ} \mathrm{C}$ & $13,41 \mathrm{bcA}$ & $16,57 \mathrm{abA}$ & $15,42 \mathrm{bcA}$ & 21 & $16,28 \mathrm{aA}$ & 19,86 aA & $17,27 \mathrm{abA}$ \\
\hline \multicolumn{5}{|c|}{ Dia 14} & \multicolumn{3}{|c|}{$15^{\circ} \mathrm{C}$} \\
\hline AMB & NA & NA & NA & 0 & $0,00 \mathrm{cA}$ & $0,00 \mathrm{cA}$ & $0,00 \mathrm{bA}$ \\
\hline $6^{\circ} \mathrm{C}$ & $16,83 \mathrm{aA}$ & $17,99 \mathrm{abA}$ & $19,99 \mathrm{aA}$ & 4 & $13,66 \mathrm{abA}$ & $13,85 \mathrm{abA}$ & $13,67 \mathrm{aA}$ \\
\hline $10^{\circ} \mathrm{C}$ & $17,76 \mathrm{aA}$ & 19,63 aA & $20,06 \mathrm{aA}$ & 7 & $12,25 \mathrm{bA}$ & $15,03 \mathrm{aA}$ & $13,98 \mathrm{aA}$ \\
\hline $15^{\circ} \mathrm{C}$ & $16,34 \mathrm{aA}$ & $11,81 \mathrm{cA}$ & $13,11 \mathrm{cA}$ & 14 & $16,34 \mathrm{aA}$ & $11,81 \mathrm{bA}$ & $13,11 \mathrm{aA}$ \\
\hline $20{ }^{\circ} \mathrm{C}$ & $15,61 \mathrm{aA}$ & $15,10 \mathrm{bA}$ & $16,17 \mathrm{bA}$ & 21 & NA & NA & NA \\
\hline \multicolumn{5}{|c|}{ Dia 21} & \multicolumn{3}{|c|}{$20^{\circ} \mathrm{C}$} \\
\hline AMB & NA & NA & NA & 0 & $0,00 \mathrm{cA}$ & $0,00 \mathrm{cA}$ & $0,00 \mathrm{cA}$ \\
\hline $6{ }^{\circ} \mathrm{C}$ & $15,10 \mathrm{aA}$ & $18,70 \mathrm{aA}$ & $18,83 \mathrm{aA}$ & 4 & $12,73 \mathrm{bA}$ & $13,51 \mathrm{bA}$ & 13,98 bA \\
\hline $10^{\circ} \mathrm{C}$ & $16,28 \mathrm{aA}$ & $19,86 \mathrm{aA}$ & $17,27 \mathrm{aA}$ & 7 & $13,41 \mathrm{abA}$ & $16,57 \mathrm{aA}$ & $15,42 \mathrm{abA}$ \\
\hline $15^{\circ} \mathrm{C}$ & NA & NA & NA & 14 & $15,61 \mathrm{aA}$ & $15,10 \mathrm{abA}$ & $16,17 \mathrm{aA}$ \\
\hline $20^{\circ} \mathrm{C}$ & NA & NA & NA & 21 & NA & NA & NA \\
\hline
\end{tabular}

TEMP: temperatura; DA: dia de análise; AMB: ambiente; HI: hipoclorito inorgânico; HO: hipoclorito orgânico; NA: Não Analisado. Letras minúsculas iguais, na mesma coluna, não diferem significativamente no nível de $5 \%$ no teste de Tukey. Letras maiúsculas iguais, na mesma linha, não diferem significativamente no nível de 5\% no teste de Tukey.

Os valores de cromaticidade (chroma) estão apresentados na Tabela 3. A cromaticidade ou chroma expressa a intensidade da cor, ou seja, a cor em termos de saturação de pigmentos. No estudo realizado, os frutos de maracujás apresentaram valores de chroma intermediários, não sendo possível afirmar que os mesmos apresentam cores neutras ou vívidas. Os valores de chroma dos frutos apresentaram oscilações entre 19,44 e 39,68 durante todo o armazenamento (Tabela 3), com variação significativa entre as condições de armazenamento somente aos
14 dias, em que o produto lavado com água apresentou menores valores de cromaticidade quando mantido na temperatura de $15^{\circ} \mathrm{C}$. Na temperatura de $20^{\circ} \mathrm{C}$, este tratamento apresentou maior valor quando comparado ao sanitizado com hipoclorito orgânico.

Durante o período de armazenamento, ocorreu variação nos valores de chroma do produto mantido nas temperaturas de $15{ }^{\circ} \mathrm{C}$ e $20^{\circ} \mathrm{C}$. Aos 14 dias de armazenamento, o produto lavado apenas com água apresentou valores inferiores de chroma, não diferindo 
Conservação pós-colheita de frutos de Passiflora setacea DC. submetidos a diferentes sanitizantes e temperaturas de armazenamento

Rinaldi, M. M. et al.

Tabela 3. Valores médios de chroma em frutos de Passiflora setacea cv. BRS Pérola do Cerrado, submetidos a diferentes tratamentos.

\begin{tabular}{|c|c|c|c|c|c|c|c|}
\hline \multirow{5}{*}{ TEMP } & \multicolumn{7}{|c|}{ Chroma } \\
\hline & \multicolumn{3}{|c|}{ DA } & \multirow{4}{*}{ DA } & \multirow{2}{*}{\multicolumn{3}{|c|}{$\begin{array}{c}\text { TEMP } \\
\text { Sanitizante }\end{array}$}} \\
\hline & \multicolumn{3}{|c|}{ Sanitizante } & & & & \\
\hline & $\mathrm{H}_{2} \mathrm{O}$ & HI & $\mathrm{HO}$ & & $\mathrm{H}_{2} \mathrm{O}$ & $\mathrm{HI}$ & $\mathrm{HO}$ \\
\hline & \multicolumn{3}{|c|}{ Dia 0} & & \multicolumn{3}{|c|}{ AMB } \\
\hline AMB & $32,90 \mathrm{aA}$ & $32,90 \mathrm{aA}$ & $32,90 \mathrm{aA}$ & 0 & $32,90 \mathrm{aA}$ & $32,90 \mathrm{aA}$ & $32,90 \mathrm{aA}$ \\
\hline $6^{\circ} \mathrm{C}$ & $32,90 \mathrm{aA}$ & $32,90 \mathrm{aA}$ & $32,90 \mathrm{aA}$ & 4 & 30,08 aA & $28,88 \mathrm{aA}$ & $28,57 \mathrm{aA}$ \\
\hline $10^{\circ} \mathrm{C}$ & $32,90 \mathrm{aA}$ & $32,90 \mathrm{aA}$ & $32,90 \mathrm{aA}$ & 7 & $26,65 \mathrm{aA}$ & $27,25 \mathrm{aA}$ & $26,14 \mathrm{aA}$ \\
\hline $15^{\circ} \mathrm{C}$ & $32,90 \mathrm{aA}$ & $32,90 \mathrm{aA}$ & $32,90 \mathrm{aA}$ & 14 & NA & NA & NA \\
\hline $20^{\circ} \mathrm{C}$ & $32,90 \mathrm{aA}$ & $32,90 \mathrm{aA}$ & $32,90 \mathrm{aA}$ & 21 & NA & NA & NA \\
\hline \multicolumn{5}{|c|}{ Dia 4} & \multicolumn{3}{|c|}{$6{ }^{\circ} \mathrm{C}$} \\
\hline AMB & 30,08 aA & 28,88 aA & 28,57 aA & 0 & $32,90 \mathrm{aA}$ & 32,90 aA & $32,90 \mathrm{aA}$ \\
\hline $6{ }^{\circ} \mathrm{C}$ & 35,38 aA & $37,19 \mathrm{aA}$ & $35,64 \mathrm{aA}$ & 4 & 35,38 aA & $37,19 \mathrm{aA}$ & $35,64 \mathrm{aA}$ \\
\hline $10^{\circ} \mathrm{C}$ & $38,29 \mathrm{aA}$ & $37,26 \mathrm{aA}$ & 35,33 aA & 7 & $35,67 \mathrm{aA}$ & 38,86 aA & $37,27 \mathrm{aA}$ \\
\hline $15^{\circ} \mathrm{C}$ & 39,68 aA & $32,81 \mathrm{aA}$ & $27,53 \mathrm{aA}$ & 14 & 35,40 aA & 34,68 aA & $33,95 \mathrm{aA}$ \\
\hline \multirow[t]{2}{*}{$20^{\circ} \mathrm{C}$} & $29,60 \mathrm{aA}$ & $25,70 \mathrm{aA}$ & 23,75 aA & 21 & $36,10 \mathrm{aA}$ & 35,09 aA & $36,37 \mathrm{aA}$ \\
\hline & \multicolumn{3}{|c|}{ Dia 7} & & \multicolumn{3}{|c|}{$10^{\circ} \mathrm{C}$} \\
\hline AMB & 26,65 aA & 27,25 aA & $26,14 \mathrm{aA}$ & 0 & $32,90 \mathrm{aA}$ & $32,90 \mathrm{aA}$ & $32,90 \mathrm{aA}$ \\
\hline $6^{\circ} \mathrm{C}$ & 35,67 aA & $38,86 \mathrm{aA}$ & 37,27 aA & 4 & 38,29 aA & 37,26 aA & $35,33 \mathrm{aA}$ \\
\hline $10^{\circ} \mathrm{C}$ & $39,00 \mathrm{aA}$ & $36,97 \mathrm{aA}$ & 37,38 aA & 7 & $39,00 \mathrm{aA}$ & 36,97 aA & $37,38 \mathrm{aA}$ \\
\hline $15^{\circ} \mathrm{C}$ & 28,79 aA & $24,89 \mathrm{aA}$ & $26,07 \mathrm{aA}$ & 14 & $34,71 \mathrm{aA}$ & 34,14 aA & $33,06 \mathrm{aA}$ \\
\hline \multirow[t]{2}{*}{$20^{\circ} \mathrm{C}$} & $27,73 \mathrm{aA}$ & $25,08 \mathrm{aA}$ & $22,37 \mathrm{aA}$ & 21 & $27,66 \mathrm{aA}$ & 27,48 aA & $30,29 \mathrm{aA}$ \\
\hline & \multicolumn{3}{|c|}{ Dia 14} & & \multicolumn{3}{|c|}{$15^{\circ} \mathrm{C}$} \\
\hline AMB & NA & NA & NA & 0 & $32,90 \mathrm{abA}$ & $32,90 \mathrm{aA}$ & 32,90 aA \\
\hline $6^{\circ} \mathrm{C}$ & $35,40 \mathrm{aA}$ & $34,68 \mathrm{aA}$ & 33,95 aA & 4 & 39,68 aA & $32,81 \mathrm{aB}$ & $27,53 \mathrm{aB}$ \\
\hline $10^{\circ} \mathrm{C}$ & 34,71 aA & 34,14 aA & 33,06 aA & 7 & $26,78 \mathrm{bcA}$ & $24,89 \mathrm{bA}$ & $26,07 \mathrm{aA}$ \\
\hline $15^{\circ} \mathrm{C}$ & $23,44 \mathrm{bB}$ & 29,68 aA & 30,42 aA & 14 & $23,44 \mathrm{cB}$ & $29,68 \mathrm{abAB}$ & $30,42 \mathrm{aA}$ \\
\hline \multirow[t]{2}{*}{$20^{\circ} \mathrm{C}$} & $28,06 \mathrm{bA}$ & $23,08 \mathrm{bAB}$ & $19,44 \mathrm{bB}$ & 21 & NA & NA & NA \\
\hline & \multicolumn{3}{|c|}{ Dia 21} & & \multicolumn{3}{|c|}{$20^{\circ} \mathrm{C}$} \\
\hline AMB & NA & NA & NA & 0 & $32,90 \mathrm{aA}$ & $32,90 \mathrm{aA}$ & $32,90 \mathrm{aA}$ \\
\hline $6{ }^{\circ} \mathrm{C}$ & $36,10 \mathrm{aA}$ & $35,09 \mathrm{aA}$ & 36,37 aA & 4 & $29,60 a b A$ & $25,70 \mathrm{bAB}$ & $23,75 \mathrm{bB}$ \\
\hline $10^{\circ} \mathrm{C}$ & 27,66 aA & 27,48 aA & 30,29 aA & 7 & $27,73 \mathrm{bA}$ & $25,08 \mathrm{bAB}$ & $22,37 \mathrm{bB}$ \\
\hline $15^{\circ} \mathrm{C}$ & NA & NA & NA & 14 & $28,06 \mathrm{bA}$ & 23,08 bB & $19,44 \mathrm{bB}$ \\
\hline $20^{\circ} \mathrm{C}$ & NA & NA & NA & 21 & NA & NA & NA \\
\hline
\end{tabular}

TEMP: temperatura; DA: dia de análise; AMB: ambiente; HI: hipoclorito inorgânico; HO: hipoclorito orgânico; NA: Não Analisado. Letras minúsculas iguais, na mesma coluna, não diferem significativamente no nível de 5\% no teste de Tukey. Letras maiúsculas iguais, na mesma linha, não diferem significativamente no nível de 5\% no teste de Tukey.

significativamente do produto sanitizado com hipoclorito orgânico.

De maneira geral, o produto mantido nas temperaturas de $6{ }^{\circ} \mathrm{C}$ e $10^{\circ} \mathrm{C}$ apresentou maiores valores de ângulo hue. Nos demais tratamentos, ocorreu redução nos valores durante 0 armazenamento. Os valores variaram entre 77,54 e 91,40 durante todo o armazenamento (Tabela 4). Ângulo hue de 90 significa cor amarela e, à medida que este valor se afasta em direção a zero, mais vermelha será a cor de fundo. No decorrer do armazenamento, o produto que apresentou menor redução nos valores de ângulo hue foi o mantido na temperatura de $6{ }^{\circ} \mathrm{C}$, indicando que a epiderme dos frutos estava mais verde. $\mathrm{Na}$ temperatura de $6^{\circ} \mathrm{C}$, o produto submetido à sanitização com hipoclorito orgânico foi o que apresentou maior redução nos valores de ângulo hue.

O maior valor $(4,85)$ de acidez titulável foi obtido no produto apenas lavado com $\mathrm{H}_{2} \mathrm{O}$ aos sete dias de armazenamento, na temperatura de $10{ }^{\circ} \mathrm{C}$ (Tabela 5). De maneira geral, o produto armazenado nas temperaturas de $6{ }^{\circ} \mathrm{C}$ e $10^{\circ} \mathrm{C}$ apresentou uma maior conservação nos valores de acidez titulável durante o armazenamento, o 
Conservação pós-colheita de frutos de Passiflora setacea DC. submetidos a diferentes sanitizantes e temperaturas de armazenamento

Rinaldi, M. M. et al.

Tabela 4. Valores médios de ângulo hue em frutos de Passiflora setacea cv. BRS Pérola do Cerrado, submetidos a diferentes tratamentos.

\begin{tabular}{|c|c|c|c|c|c|c|c|}
\hline \multirow{5}{*}{ TEMP } & \multicolumn{7}{|c|}{ Ângulo hue } \\
\hline & \multicolumn{3}{|c|}{ DA } & \multirow{4}{*}{ DA } & \multicolumn{3}{|c|}{ TEMP } \\
\hline & \multicolumn{3}{|c|}{ Sanitizante } & & \multicolumn{3}{|c|}{ Sanitizante } \\
\hline & $\mathrm{H}_{2} \mathrm{O}$ & $\mathrm{HI}$ & $\mathrm{HO}$ & & $\mathrm{H}_{2} \mathrm{O}$ & HI & HO \\
\hline & \multicolumn{3}{|c|}{ Dia 0} & & \multicolumn{3}{|c|}{ AMB } \\
\hline AMB & 90,50 aA & 90,50 aA & 90,50 aA & 0 & 90,50 aA & 90,50 aA & 90,50 aA \\
\hline $6^{\circ} \mathrm{C}$ & $90,50 \mathrm{aA}$ & $90,50 \mathrm{aA}$ & $90,50 \mathrm{aA}$ & 4 & $89,07 \mathrm{aA}$ & $87,74 \mathrm{aA}$ & $86,56 \mathrm{aA}$ \\
\hline $10^{\circ} \mathrm{C}$ & 90,50 aA & $90,50 \mathrm{aA}$ & 90,50 aA & 7 & $84,27 \mathrm{aA}$ & $85,03 \mathrm{aA}$ & $82,59 \mathrm{aA}$ \\
\hline $15^{\circ} \mathrm{C}$ & $90,50 \mathrm{aA}$ & $90,50 \mathrm{aA}$ & $90,50 \mathrm{aA}$ & 14 & NA & NA & NA \\
\hline $20^{\circ} \mathrm{C}$ & $90,50 \mathrm{aA}$ & 90,50 aA & $90,50 \mathrm{aA}$ & 21 & NA & NA & NA \\
\hline \multicolumn{5}{|c|}{ Dia 4} & \multicolumn{3}{|c|}{$6{ }^{\circ} \mathrm{C}$} \\
\hline AMB & $88,04 \mathrm{abA}$ & $87,74 \mathrm{bcAB}$ & $86,56 \mathrm{bcB}$ & 0 & $90,50 \mathrm{aA}$ & $90,50 \mathrm{aA}$ & 90,50 aA \\
\hline $6^{\circ} \mathrm{C}$ & $91,40 \mathrm{aA}$ & $90,45 \mathrm{abA}$ & $89,13 \mathrm{abA}$ & 4 & $91,40 \mathrm{aA}$ & 90,45 aAB & $89,13 \mathrm{abB}$ \\
\hline $10^{\circ} \mathrm{C}$ & $91,06 \mathrm{abA}$ & $90,04 \mathrm{abA}$ & $90,01 \mathrm{aA}$ & 7 & $90,50 \mathrm{aA}$ & 89,83 aA & $90,90 \mathrm{aA}$ \\
\hline $15^{\circ} \mathrm{C}$ & $88,48 \mathrm{bAB}$ & 90,80 aA & $88,05 \mathrm{abcB}$ & 14 & 89,98 aA & 89,26 aA & $89,63 \mathrm{aA}$ \\
\hline $20^{\circ} \mathrm{C}$ & 89,69 abA & $85,64 \mathrm{cB}$ & $86,07 \mathrm{cB}$ & 21 & $87,82 \mathrm{bAB}$ & 89,28 aA & $87,53 \mathrm{bB}$ \\
\hline \multicolumn{5}{|c|}{ Dia 7} & \multicolumn{3}{|c|}{$10^{\circ} \mathrm{C}$} \\
\hline $\mathrm{AMB}$ & $84,27 \mathrm{bA}$ & 85,03 bA & $82,59 \mathrm{cA}$ & 0 & $90,50 \mathrm{aA}$ & $90,50 \mathrm{aA}$ & $90,50 \mathrm{aA}$ \\
\hline $6^{\circ} \mathrm{C}$ & $90,50 \mathrm{aA}$ & 89,83 aA & 90,90 aA & 4 & $91,06 \mathrm{aA}$ & $90,04 \mathrm{aA}$ & $90,01 \mathrm{aA}$ \\
\hline $10^{\circ} \mathrm{C}$ & $90,05 \mathrm{aA}$ & $90,12 \mathrm{aA}$ & $89,56 \mathrm{abA}$ & 7 & $90,05 \mathrm{aA}$ & $90,12 \mathrm{aA}$ & 90,56 aA \\
\hline $15^{\circ} \mathrm{C}$ & 85,09 bA & 83,76 bA & $85,63 \mathrm{bcA}$ & 14 & 87,59 aA & 85,93 aA & 86,59 aA \\
\hline $20^{\circ} \mathrm{C}$ & $87,23 \mathrm{abA}$ & $78,69 \mathrm{cC}$ & $83,19 \mathrm{cB}$ & 21 & $83,61 \mathrm{aA}$ & $77,54 \mathrm{aA}$ & $83,93 \mathrm{aA}$ \\
\hline \multicolumn{5}{|c|}{ Dia 14} & \multicolumn{3}{|c|}{$15^{\circ} \mathrm{C}$} \\
\hline AMB & NA & NA & NA & 0 & 90,50 aA & 90,50 aA & $90,50 \mathrm{aA}$ \\
\hline $6^{\circ} \mathrm{C}$ & 89,98 aA & 89,26 aA & $89,63 \mathrm{aA}$ & 4 & $88,48 \mathrm{abA}$ & 90,80 aA & $88,05 a b A$ \\
\hline $10^{\circ} \mathrm{C}$ & $87,59 \mathrm{aA}$ & $85,93 \mathrm{abA}$ & $86,59 \mathrm{abA}$ & 7 & $85,09 \mathrm{bA}$ & $83,76 \mathrm{bA}$ & $85,63 \mathrm{bA}$ \\
\hline $15^{\circ} \mathrm{C}$ & $79,11 \mathrm{bB}$ & 87,38 aA & 84,62 bA & 14 & $79,11 \mathrm{cB}$ & $87,38 \mathrm{abA}$ & 84,62 bA \\
\hline $20^{\circ} \mathrm{C}$ & $81,16 \mathrm{bA}$ & 81,93 bA & $78,83 \mathrm{cA}$ & 21 & NA & NA & NA \\
\hline \multicolumn{5}{|c|}{ Dia 21} & \multicolumn{3}{|c|}{$20^{\circ} \mathrm{C}$} \\
\hline AMB & NA & $N A$ & NA & 0 & $90,50 \mathrm{aA}$ & $90,50 \mathrm{aA}$ & $90,50 \mathrm{aA}$ \\
\hline $6^{\circ} \mathrm{C}$ & $87,82 \mathrm{aA}$ & 89,28 aA & 87,53 aA & 4 & 89,69 aA & $85,64 \mathrm{bA}$ & $86,07 \mathrm{abA}$ \\
\hline $10^{\circ} \mathrm{C}$ & $83,61 \mathrm{aA}$ & $77,54 \mathrm{aA}$ & 83,93 aA & 7 & 87,23 aA & $78,69 \mathrm{cB}$ & $83,19 \mathrm{bcA}$ \\
\hline $15^{\circ} \mathrm{C}$ & NA & NA & NA & 14 & $81,16 \mathrm{bA}$ & $81,93 \mathrm{bcA}$ & $78,83 \mathrm{cA}$ \\
\hline $20^{\circ} \mathrm{C}$ & NA & NA & NA & 21 & NA & NA & NA \\
\hline
\end{tabular}

TEMP: temperatura; DA: dia de análise; AMB: ambiente; HI: hipoclorito inorgânico; HO: hipoclorito orgânico; NA: Não Analisado. Letras minúsculas iguais, na mesma coluna, não diferem significativamente no nível de $5 \%$ no teste de Tukey. Letras maiúsculas iguais, na mesma linha, não diferem significativamente no nível de $5 \%$ no teste de Tukey.

que já era esperado, uma vez que a redução da acidez é maior em frutos mantidos em temperaturas mais altas, devido à maior atividade metabólica (ARRUDA et al., 2011). O produto mantido a $6{ }^{\circ} \mathrm{C}$ e $10{ }^{\circ} \mathrm{C}$ também manteve por mais tempo os valores de acidez titulável acima do limite mínimo (2,5 g $\left.100 \mathrm{~g}^{-1}\right)$ permitido pela legislação brasileira, para sucos de maracujás.

Do ponto de vista industrial, o alto teor de acidez é interessante, pois reduz a necessidade de se adicionarem acidulantes e melhora a qualidade nutricional, a segurança alimentar e o aspecto sensorial (PITA, 2012). Não ocorreu redução linear na acidez dos frutos de Passiflora setacea durante o armazenamento (Tabela 5), contrariando o observado por Silva et al. (2009), em frutos de maracujáamarelo.

Com exceção do produto sanitizado com hipoclorito inorgânico e armazenado na temperatura de $20{ }^{\circ} \mathrm{C}$ aos 14 dias de armazenamento, todos os demais valores de sólidos solúveis obtidos foram superiores ao teor mínimo exigido pelo Ministério da Agricultura (11 $\left.{ }^{\circ} \mathrm{Brix}\right)$, para o 
Conservação pós-colheita de frutos de Passiflora setacea DC. submetidos a diferentes sanitizantes e temperaturas de armazenamento

Rinaldi, M. M. et al.

Tabela 5. Valores médios de acidez titulável em frutos de Passiflora setacea cv. BRS Pérola do Cerrado, submetidos a diferentes tratamentos.

\begin{tabular}{|c|c|c|c|c|c|c|c|}
\hline \multirow{5}{*}{ TEMP } & \multicolumn{7}{|c|}{ Acidez titulável (g de ácido cítrico/100 g de produto) } \\
\hline & \multicolumn{3}{|c|}{ DA } & \multirow{4}{*}{ DA } & \multicolumn{3}{|c|}{ TEMP } \\
\hline & \multicolumn{3}{|c|}{ Sanitizante } & & \multicolumn{3}{|c|}{ Sanitizante } \\
\hline & $\mathrm{H}_{2} \mathrm{O}$ & HI & HO & & $\mathrm{H}_{2} \mathrm{O}$ & HI & HO \\
\hline & \multicolumn{3}{|c|}{ Dia 0} & & \multicolumn{3}{|c|}{ AMB } \\
\hline AMB & $2,73 \mathrm{aA}$ & 2,73 aA & $2,73 \mathrm{aA}$ & 0 & $2,73 \mathrm{aA}$ & $2,73 \mathrm{bA}$ & $2,73 \mathrm{bA}$ \\
\hline $6^{\circ} \mathrm{C}$ & 2,73 aA & $2,73 \mathrm{aA}$ & $2,73 \mathrm{aA}$ & 4 & $1,81 \mathrm{cB}$ & $1,64 \mathrm{cC}$ & $2,48 \mathrm{cA}$ \\
\hline $10^{\circ} \mathrm{C}$ & 2,73 aA & $2,73 \mathrm{aA}$ & $2,73 \mathrm{aA}$ & 7 & $2,17 \mathrm{bB}$ & 2,88 aA & $2,84 \mathrm{aA}$ \\
\hline $15^{\circ} \mathrm{C}$ & $2,73 \mathrm{aA}$ & $2,73 \mathrm{aA}$ & $2,73 \mathrm{aA}$ & 14 & NA & NA & NA \\
\hline $20^{\circ} \mathrm{C}$ & $2,73 \mathrm{aA}$ & $2,73 \mathrm{aA}$ & $2,73 \mathrm{aA}$ & 21 & NA & NA & NA \\
\hline \multicolumn{5}{|c|}{ Dia 4} & \multicolumn{3}{|c|}{$6^{\circ} \mathrm{C}$} \\
\hline $\mathrm{AMB}$ & $1,81 \mathrm{cB}$ & $1,64 \mathrm{dC}$ & $2,48 \mathrm{bA}$ & 0 & $2,73 \mathrm{cA}$ & $2,73 \mathrm{cA}$ & $2,73 \mathrm{cA}$ \\
\hline $6{ }^{\circ} \mathrm{C}$ & $2,95 \mathrm{aA}$ & $3,00 \mathrm{abA}$ & $3,04 \mathrm{aA}$ & 4 & $2,95 \mathrm{bA}$ & $3,00 \mathrm{bA}$ & $3,04 \mathrm{bA}$ \\
\hline $10^{\circ} \mathrm{C}$ & $2,82 \mathrm{abB}$ & $3,15 \mathrm{aA}$ & $3,14 \mathrm{aA}$ & 7 & $4,16 \mathrm{aC}$ & $4,57 \mathrm{aB}$ & $4,84 \mathrm{aA}$ \\
\hline $15^{\circ} \mathrm{C}$ & $2,73 \mathrm{bB}$ & $2,91 \mathrm{bA}$ & $2,27 \mathrm{cC}$ & 14 & $2,67 \mathrm{cA}$ & $2,64 \mathrm{cdA}$ & $2,41 \mathrm{~dB}$ \\
\hline $20^{\circ} \mathrm{C}$ & $1,86 \mathrm{cB}$ & $1,99 \mathrm{cB}$ & $2,28 \mathrm{cA}$ & 21 & $2,70 \mathrm{cB}$ & $2,53 \mathrm{dC}$ & $3,05 \mathrm{bA}$ \\
\hline \multicolumn{5}{|c|}{ Dia 7} & \multicolumn{3}{|c|}{$10^{\circ} \mathrm{C}$} \\
\hline AMB & $2,17 \mathrm{cB}$ & $2,88 \mathrm{cA}$ & $2,84 \mathrm{bA}$ & 0 & $2,73 \mathrm{bA}$ & $2,73 \mathrm{cA}$ & $2,73 \mathrm{cA}$ \\
\hline $6^{\circ} \mathrm{C}$ & $4,16 \mathrm{bC}$ & $4,57 \mathrm{bB}$ & $4,84 \mathrm{aA}$ & 4 & $2,82 \mathrm{bB}$ & $3,15 \mathrm{bA}$ & $3,14 \mathrm{bA}$ \\
\hline $10^{\circ} \mathrm{C}$ & $4,85 \mathrm{aA}$ & 4,78 aA & $4,72 \mathrm{aA}$ & 7 & $4,85 \mathrm{aA}$ & 4,78 aA & $4,72 \mathrm{aA}$ \\
\hline $15^{\circ} \mathrm{C}$ & $2,16 \mathrm{cB}$ & $2,78 \mathrm{cA}$ & $2,11 \mathrm{cB}$ & 14 & $2,03 \mathrm{cB}$ & $2,66 \mathrm{cA}$ & $1,95 \mathrm{~dB}$ \\
\hline $20^{\circ} \mathrm{C}$ & $2,13 \mathrm{cA}$ & $2,03 \mathrm{dA}$ & $2,16 \mathrm{cA}$ & 21 & $2,90 \mathrm{bA}$ & $2,35 \mathrm{dC}$ & $2,63 \mathrm{cB}$ \\
\hline \multicolumn{5}{|c|}{ Dia 14} & \multicolumn{3}{|c|}{$15^{\circ} \mathrm{C}$} \\
\hline AMB & NA & NA & NA & 0 & 2,73 aA & $2,73 \mathrm{bA}$ & $2,73 \mathrm{aA}$ \\
\hline $6^{\circ} \mathrm{C}$ & $2,67 \mathrm{aA}$ & $2,64 \mathrm{aA}$ & $2,41 \mathrm{aB}$ & 4 & $2,73 \mathrm{aB}$ & $2,91 \mathrm{aA}$ & $2,27 \mathrm{cC}$ \\
\hline $10^{\circ} \mathrm{C}$ & $2,03 \mathrm{cB}$ & $2,66 \mathrm{aA}$ & $1,95 \mathrm{bB}$ & 7 & $2,16 \mathrm{bB}$ & $2,78 \mathrm{abA}$ & $2,11 \mathrm{~dB}$ \\
\hline $15^{\circ} \mathrm{C}$ & $2,00 \mathrm{cB}$ & $2,29 \mathrm{bA}$ & $2,44 \mathrm{aA}$ & 14 & $2,00 \mathrm{cC}$ & $2,29 \mathrm{cB}$ & $2,44 \mathrm{bA}$ \\
\hline $20^{\circ} \mathrm{C}$ & $2,39 \mathrm{bA}$ & $1,93 \mathrm{cB}$ & $2,02 \mathrm{bB}$ & 21 & NA & NA & NA \\
\hline \multicolumn{5}{|c|}{ Dia 21} & \multicolumn{3}{|c|}{$20^{\circ} \mathrm{C}$} \\
\hline AMB & NA & NA & NA & 0 & $2,73 \mathrm{aA}$ & 2,73 aA & 2,73 aA \\
\hline $6^{\circ} \mathrm{C}$ & 2,70 bB & $2,53 \mathrm{aC}$ & $3,05 \mathrm{aA}$ & 4 & $1,86 \mathrm{~dB}$ & 1,99 bB & 2,28 bA \\
\hline $10^{\circ} \mathrm{C}$ & $2,90 \mathrm{aA}$ & $2,35 \mathrm{bC}$ & $2,63 \mathrm{bB}$ & 7 & $2,13 \mathrm{cA}$ & $2,03 \mathrm{bA}$ & $2,16 \mathrm{bcA}$ \\
\hline $15^{\circ} \mathrm{C}$ & NA & NA & NA & 14 & $2,39 \mathrm{bA}$ & $1,93 \mathrm{bB}$ & $2,02 \mathrm{cB}$ \\
\hline $20^{\circ} \mathrm{C}$ & NA & NA & NA & 21 & NA & NA & NA \\
\hline
\end{tabular}

TEMP: temperatura; DA: dia de análise; AMB: ambiente; HI: hipoclorito inorgânico; HO: hipoclorito orgânico; NA: Não Analisado. Letras minúsculas iguais, na mesma coluna, não diferem significativamente no nível de $5 \%$ no teste de Tukey. Letras maiúsculas iguais, na mesma linha, não diferem significativamente no nível de $5 \%$ no teste de Tukey.

suco in natura (BRASIL, 2000). Além disso, a matéria-prima e o produto armazenado, na maioria dos períodos de análise, corresponderam ao requerido pela indústria de processamento de sucos (14ㅁix) (Tabela 6), em que há preferência por frutos com maior teor de sólidos solúveis.

Os valores de sólidos solúveis oscilaram entre 10,60 ${ }^{\circ}$ Brix e 15,37 ${ }^{\circ}$ Brix, com valor inicial de $15,10^{\circ}$ Brix (Tabela 6), em que as maiores oscilações ocorreram em frutos mantidos à temperatura ambiente, $15^{\circ} \mathrm{C} \mathrm{e} 20^{\circ} \mathrm{C}$. Para a agroindústria, é interessante que os frutos apresentem elevados teores de sólidos solúveis (> $13^{\circ} \mathrm{Brix}$ ), sendo esse aspecto considerado um indicador de qualidade do fruto (BRUCKNER et al., 2002).

Decréscimo linear nos valores de sólidos solúveis foi observado em frutos de maracujazeiro-amarelo variedade FB-200 para consumo in natura, durante o armazenamento pós-colheita em temperatura de $21 \pm 2{ }^{\circ} \mathrm{C}$ e umidade relativa do ar de $70 \%$, por 16 dias (VENÂNCIO et al., 2013). As oscilações nos valores de sólidos solúveis durante o armazenamento provavelmente devem-se às 
Conservação pós-colheita de frutos de Passiflora setacea DC. submetidos a diferentes sanitizantes e temperaturas de armazenamento

Rinaldi, M. M. et al.

Tabela 6. Valores médios de sólidos solúveis em frutos de Passiflora setacea cv. BRS Pérola do Cerrado, submetidos a diferentes tratamentos.

\begin{tabular}{|c|c|c|c|c|c|c|c|}
\hline \multirow{5}{*}{ TEMP } & \multicolumn{7}{|c|}{ Sólidos solúveis ( ${ }^{\circ}$ Brix) } \\
\hline & \multicolumn{3}{|c|}{ DA } & \multirow{4}{*}{ DA } & \multicolumn{3}{|c|}{ TEMP } \\
\hline & \multicolumn{3}{|c|}{ Sanitizante } & & \multicolumn{3}{|c|}{ Sanitizante } \\
\hline & $\mathrm{H}_{2} \mathrm{O}$ & $\mathrm{HI}$ & HO & & $\mathrm{H}_{2} \mathrm{O}$ & HI & HO \\
\hline & \multicolumn{3}{|c|}{ Dia 0} & & \multicolumn{3}{|c|}{ AMB } \\
\hline AMB & $15,10 \mathrm{aA}$ & $15,10 \mathrm{aA}$ & $15,10 \mathrm{aA}$ & 0 & $15,10 \mathrm{aA}$ & $15,10 \mathrm{aA}$ & $15,10 \mathrm{aA}$ \\
\hline $6^{\circ} \mathrm{C}$ & $15,10 \mathrm{aA}$ & $15,10 \mathrm{aA}$ & $15,10 \mathrm{aA}$ & 4 & $12,42 \mathrm{cB}$ & $11,58 \mathrm{cC}$ & $14,13 \mathrm{bA}$ \\
\hline $10{ }^{\circ} \mathrm{C}$ & 15,10 aA & 15,10 aA & $15,10 \mathrm{aA}$ & 7 & $13,53 \mathrm{bC}$ & $14,68 \mathrm{bA}$ & $14,23 \mathrm{bB}$ \\
\hline $15^{\circ} \mathrm{C}$ & $15,10 \mathrm{aA}$ & $15,10 \mathrm{aA}$ & $15,10 \mathrm{aA}$ & 14 & NA & NA & NA \\
\hline $20^{\circ} \mathrm{C}$ & $15,10 \mathrm{aA}$ & 15,10 aA & $15,10 \mathrm{aA}$ & 21 & NA & NA & NA \\
\hline \multicolumn{5}{|c|}{ Dia 4} & \multicolumn{3}{|c|}{$6^{\circ} \mathrm{C}$} \\
\hline AMB & $12,42 \mathrm{eB}$ & $11,58 \mathrm{dC}$ & $14,13 \mathrm{bcA}$ & 0 & $15,10 \mathrm{aA}$ & $15,10 \mathrm{aA}$ & $15,10 \mathrm{aA}$ \\
\hline $6^{\circ} \mathrm{C}$ & $15,33 \mathrm{aA}$ & $14,27 \mathrm{bB}$ & $14,47 \mathrm{bB}$ & 4 & $15,33 \mathrm{aA}$ & $14,27 \mathrm{bB}$ & $14,47 \mathrm{bB}$ \\
\hline $10^{\circ} \mathrm{C}$ & $14,63 \mathrm{bA}$ & $13,47 \mathrm{cB}$ & $13,67 \mathrm{~dB}$ & 7 & $14,53 \mathrm{bB}$ & $15,37 \mathrm{aA}$ & $14,29 \mathrm{bB}$ \\
\hline $15^{\circ} \mathrm{C}$ & $13,47 \mathrm{~dB}$ & $11,97 \mathrm{dC}$ & $14,00 \mathrm{cdA}$ & 14 & $15,33 \mathrm{aA}$ & 14,43 bB & $15,17 \mathrm{aA}$ \\
\hline $20^{\circ} \mathrm{C}$ & $13,93 \mathrm{cB}$ & $15,23 \mathrm{aA}$ & $15,23 \mathrm{aA}$ & 21 & $13,87 \mathrm{cA}$ & $13,00 \mathrm{cB}$ & $13,37 \mathrm{cB}$ \\
\hline \multicolumn{5}{|c|}{ Dia 7} & \multicolumn{3}{|c|}{$10^{\circ} \mathrm{C}$} \\
\hline AMB & $13,53 \mathrm{dC}$ & $14,68 \mathrm{bA}$ & $14,23 \mathrm{aB}$ & 0 & $15,10 \mathrm{aA}$ & $15,10 \mathrm{aA}$ & $15,10 \mathrm{aA}$ \\
\hline $6^{\circ} \mathrm{C}$ & $14,53 \mathrm{bB}$ & $15,37 \mathrm{aA}$ & $14,29 \mathrm{aB}$ & 4 & $14,63 \mathrm{cA}$ & $13,47 \mathrm{cB}$ & $13,67 \mathrm{cB}$ \\
\hline $10^{\circ} \mathrm{C}$ & $15,30 \mathrm{aA}$ & 14,43 bB & $13,80 \mathrm{bC}$ & 7 & $15,30 \mathrm{aA}$ & 14,43 bB & $13,80 \mathrm{cC}$ \\
\hline $15^{\circ} \mathrm{C}$ & $14,20 \mathrm{cB}$ & $15,23 \mathrm{aA}$ & $14,13 \mathrm{aB}$ & 14 & $14,70 \mathrm{bcA}$ & $14,50 \mathrm{bAB}$ & $14,33 \mathrm{bB}$ \\
\hline $20^{\circ} \mathrm{C}$ & $13,77 \mathrm{dA}$ & $12,07 \mathrm{cC}$ & $12,63 \mathrm{cB}$ & 21 & $15,00 \mathrm{abB}$ & $12,97 \mathrm{dC}$ & $15,33 \mathrm{aA}$ \\
\hline \multicolumn{5}{|c|}{ Dia 14} & \multicolumn{3}{|c|}{$15^{\circ} \mathrm{C}$} \\
\hline AMB & NA & NA & NA & 0 & $15,10 \mathrm{aA}$ & $15,10 \mathrm{aA}$ & $15,10 \mathrm{aA}$ \\
\hline $6^{\circ} \mathrm{C}$ & $15,33 \mathrm{aA}$ & $14,43 \mathrm{aB}$ & $15,17 \mathrm{aA}$ & 4 & $13,47 \mathrm{cB}$ & $11,97 \mathrm{cC}$ & $14,00 \mathrm{bA}$ \\
\hline $10^{\circ} \mathrm{C}$ & $14,70 \mathrm{bA}$ & $14,50 \mathrm{aAB}$ & 14,33 bB & 7 & $14,20 \mathrm{Bb}$ & 15,23 aA & $14,13 \mathrm{bB}$ \\
\hline $15^{\circ} \mathrm{C}$ & $13,43 \mathrm{cA}$ & $13,57 \mathrm{bA}$ & $12,43 \mathrm{cB}$ & 14 & $13,43 \mathrm{cA}$ & $13,57 \mathrm{bA}$ & $12,43 \mathrm{cB}$ \\
\hline $20^{\circ} \mathrm{C}$ & $13,10 \mathrm{cA}$ & $10,60 \mathrm{cC}$ & $12,43 \mathrm{cB}$ & 21 & NA & NA & NA \\
\hline \multicolumn{5}{|c|}{ Dia 21} & \multicolumn{3}{|c|}{$20^{\circ} \mathrm{C}$} \\
\hline AMB & NA & NA & NA & 0 & $15,10 \mathrm{aA}$ & $15,10 \mathrm{aA}$ & $15,10 \mathrm{aA}$ \\
\hline $6^{\circ} \mathrm{C}$ & $13,87 \mathrm{bA}$ & $13,00 \mathrm{aB}$ & 13,37 bB & 4 & 13,93 bB & $15,23 \mathrm{aA}$ & $15,23 \mathrm{aA}$ \\
\hline $10^{\circ} \mathrm{C}$ & $15,00 \mathrm{aA}$ & $12,97 \mathrm{aB}$ & 15,33 aA & 7 & $13,77 \mathrm{bA}$ & $12,07 \mathrm{bC}$ & $12,63 \mathrm{bB}$ \\
\hline $15^{\circ} \mathrm{C}$ & NA & NA & NA & 14 & $13,10 \mathrm{cA}$ & $10,60 \mathrm{cC}$ & $12,43 \mathrm{bB}$ \\
\hline $20^{\circ} \mathrm{C}$ & NA & NA & NA & 21 & NA & NA & NA \\
\hline
\end{tabular}

TEMP: temperatura; DA: dia de análise; AMB: ambiente; HI: hipoclorito inorgânico; HO: hipoclorito orgânico; NA: Não Analisado. Letras minúsculas iguais, na mesma coluna, não diferem significativamente no nível de $5 \%$ no teste de Tukey. Letras maiúsculas iguais, na mesma linha, não diferem significativamente no nível de $5 \%$ no teste de Tukey.

características da matéria-prima, à atividade respiratória do produto e também à excessiva perda de massa fresca dos frutos ocorrida durante todo o armazenamento.

Em frutos da mesma espécie, pode ocorrer variação significativa nos valores de sólidos solúveis, sendo que, na literatura, são encontradas quantidades de sólidos solúveis para o maracujá amarelo/convencional de 13,8 ${ }^{\circ}$ Brix (MACHADO et al., 2003), 13,5 ${ }^{\circ}$ Brix e 15,4 ${ }^{\circ}$ Brix (AMARO; MONTEIRO, 2001) e $12,78^{\circ}$ Brix (PITA, 2012). Ainda em maracujá amarelo, Gomes et al. (2006) obtiveram valores de $13,72^{\circ}$ Brix e $14,88^{\circ}$ Brix para sólidos solúveis. Raimundo et al. (2009), com o mesmo tipo de amostra, obtiveram valores que variaram de 9,03 a 13,10 $\mathrm{Brix}$.

O valor médio de ratio foi de 5,48 , sendo este considerado como frutos de sabor excelente (Tabela 7 ). Os valores variaram entre 2,92 e 7,66, correspondendo às oscilações ocorridas nos sólidos solúveis (Tabela 6) e na acidez titulável (Tabela 5), durante o armazenamento. A relação entre o teor de sólidos solúveis e a acidez titulável (SS/AT) é mais representativa da percepção sensorial do 
Conservação pós-colheita de frutos de Passiflora setacea DC. submetidos a diferentes sanitizantes e temperaturas de armazenamento

Rinaldi, M. M. et al.

Tabela 7. Valores médios de ratio em frutos de Passiflora setacea cv. BRS Pérola do Cerrado, submetidos a diferentes tratamentos.

\begin{tabular}{|c|c|c|c|c|c|c|c|}
\hline \multirow{5}{*}{ TEMP } & \multicolumn{7}{|c|}{ ratio } \\
\hline & \multicolumn{3}{|c|}{ DA } & \multirow{4}{*}{ DA } & \multicolumn{3}{|c|}{ TEMP } \\
\hline & & Sanitizante & & & \multicolumn{3}{|c|}{ Sanitizante } \\
\hline & $\mathrm{H}_{2} \mathrm{O}$ & $\mathrm{HI}$ & $\mathrm{HO}$ & & $\mathrm{H}_{2} \mathrm{O}$ & HI & $\mathrm{HO}$ \\
\hline & \multicolumn{3}{|c|}{ Dia 0} & & \multicolumn{3}{|c|}{ AMB } \\
\hline AMB & 5,53 aA & $5,53 \mathrm{aA}$ & 5,53 aA & 0 & $5,53 \mathrm{cA}$ & $5,53 \mathrm{cA}$ & $5,53 \mathrm{abA}$ \\
\hline $6^{\circ} \mathrm{C}$ & 5,53 aA & $5,53 \mathrm{aA}$ & $5,53 \mathrm{aA}$ & 4 & $6,90 \mathrm{aA}$ & $7,02 \mathrm{aA}$ & $5,69 \mathrm{aB}$ \\
\hline $10^{\circ} \mathrm{C}$ & $5,53 \mathrm{aA}$ & $5,53 \mathrm{aA}$ & $5,53 \mathrm{aA}$ & 7 & $6,60 \mathrm{bA}$ & $5,82 \mathrm{bB}$ & $5,31 \mathrm{bC}$ \\
\hline $15^{\circ} \mathrm{C}$ & $5,53 \mathrm{aA}$ & $5,53 \mathrm{aA}$ & $5,53 \mathrm{aA}$ & 14 & NA & NA & NA \\
\hline $20^{\circ} \mathrm{C}$ & $5,53 \mathrm{aA}$ & $5,53 \mathrm{aA}$ & $5,53 \mathrm{aA}$ & 21 & NA & NA & NA \\
\hline \multicolumn{4}{|c|}{ Dia 4} & & \multicolumn{3}{|c|}{$6^{\circ} \mathrm{C}$} \\
\hline AMB & $6,90 \mathrm{bA}$ & $7,02 \mathrm{bA}$ & $5,69 \mathrm{bB}$ & 0 & $5,53 \mathrm{aA}$ & $5,53 \mathrm{aA}$ & $5,53 \mathrm{bA}$ \\
\hline $6^{\circ} \mathrm{C}$ & $5,20 \mathrm{cA}$ & $4,76 \mathrm{cB}$ & $4,76 \mathrm{cB}$ & 4 & $5,20 \mathrm{bA}$ & $4,76 \mathrm{cB}$ & $4,76 \mathrm{cB}$ \\
\hline $10^{\circ} \mathrm{C}$ & $5,21 \mathrm{cA}$ & $4,28 \mathrm{cdB}$ & $4,35 \mathrm{cB}$ & 7 & $3,49 \mathrm{cA}$ & $3,36 \mathrm{dA}$ & $2,95 \mathrm{eB}$ \\
\hline $15^{\circ} \mathrm{C}$ & $4,93 \mathrm{cB}$ & $4,11 \mathrm{dC}$ & $6,17 \mathrm{bA}$ & 14 & $5,75 \mathrm{aB}$ & $5,47 \mathrm{aC}$ & $6,30 \mathrm{aA}$ \\
\hline $20^{\circ} \mathrm{C}$ & $7,51 \mathrm{aA}$ & $7,66 \mathrm{aA}$ & $6,69 \mathrm{aB}$ & 21 & $5,14 \mathrm{bA}$ & $5,14 \mathrm{bA}$ & $4,38 \mathrm{~dB}$ \\
\hline \multicolumn{4}{|c|}{ Dia 7} & \multicolumn{4}{|c|}{$10^{\circ} \mathrm{C}$} \\
\hline AMB & 6,60 aA & $5,82 \mathrm{abB}$ & $5,32 \mathrm{cC}$ & 0 & $5,53 \mathrm{bA}$ & $5,53 \mathrm{aA}$ & $5,53 \mathrm{bA}$ \\
\hline $6^{\circ} \mathrm{C}$ & $3,49 \mathrm{bA}$ & $3,36 \mathrm{cA}$ & $2,95 \mathrm{~dB}$ & 4 & $5,21 \mathrm{bA}$ & $4,28 \mathrm{bB}$ & $4,35 \mathrm{cB}$ \\
\hline $10^{\circ} \mathrm{C}$ & $3,16 \mathrm{bA}$ & $3,02 \mathrm{cA}$ & $2,92 \mathrm{dA}$ & 7 & $3,16 \mathrm{cA}$ & $3,02 \mathrm{cA}$ & $2,92 \mathrm{dA}$ \\
\hline $15^{\circ} \mathrm{C}$ & $6,59 \mathrm{aA}$ & $5,49 \mathrm{bB}$ & $6,69 \mathrm{aA}$ & 14 & $7,25 \mathrm{aA}$ & $5,46 \mathrm{aB}$ & $7,36 \mathrm{aA}$ \\
\hline $20^{\circ} \mathrm{C}$ & 6,48 aA & $5,95 \mathrm{aB}$ & $5,87 \mathrm{bB}$ & 21 & $5,17 \mathrm{bB}$ & $5,52 \mathrm{aAB}$ & $5,83 \mathrm{bA}$ \\
\hline \multicolumn{4}{|c|}{ Dia 14} & & \multicolumn{3}{|c|}{$15^{\circ} \mathrm{C}$} \\
\hline AMB & NA & NA & NA & 0 & $5,53 \mathrm{bA}$ & $5,53 \mathrm{bA}$ & $5,53 \mathrm{cA}$ \\
\hline $6^{\circ} \mathrm{C}$ & $5,75 \mathrm{bB}$ & $5,47 \mathrm{aB}$ & $6,30 \mathrm{bA}$ & 4 & $4,93 \mathrm{cB}$ & $4,11 \mathrm{cC}$ & $6,17 \mathrm{bA}$ \\
\hline $10^{\circ} \mathrm{C}$ & $7,25 \mathrm{aA}$ & $5,46 \mathrm{aB}$ & 7,36 aA & 7 & $6,59 \mathrm{aA}$ & $5,49 \mathrm{bB}$ & 6,69 aA \\
\hline $15^{\circ} \mathrm{C}$ & $6,72 \mathrm{aA}$ & $5,94 \mathrm{Ab}$ & $5,10 \mathrm{cC}$ & 14 & 6,72 aA & $5,94 \mathrm{aB}$ & $5,10 \mathrm{dC}$ \\
\hline $20^{\circ} \mathrm{C}$ & $5,48 \mathrm{bB}$ & $5,50 \mathrm{aB}$ & $6,17 \mathrm{bA}$ & 21 & NA & NA & NA \\
\hline \multicolumn{4}{|c|}{ Dia 21} & & \multicolumn{3}{|c|}{$20^{\circ} \mathrm{C}$} \\
\hline AMB & NA & NA & NA & 0 & $5,53 \mathrm{cA}$ & $5,53 \mathrm{bA}$ & $5,53 \mathrm{cA}$ \\
\hline $6^{\circ} \mathrm{C}$ & $5,14 \mathrm{aA}$ & $5,14 \mathrm{bA}$ & $4,38 \mathrm{bB}$ & 4 & $7,51 \mathrm{aA}$ & $7,66 \mathrm{aA}$ & $6,69 \mathrm{aB}$ \\
\hline $10^{\circ} \mathrm{C}$ & $5,17 \mathrm{aC}$ & $5,52 \mathrm{aB}$ & $5,83 a A$ & 7 & $6,48 \mathrm{bA}$ & $5,95 \mathrm{bB}$ & $5,87 \mathrm{bcB}$ \\
\hline $15^{\circ} \mathrm{C}$ & NA & NA & NA & 14 & $5,48 \mathrm{cB}$ & $5,50 \mathrm{bB}$ & $6,17 \mathrm{abA}$ \\
\hline $20^{\circ} \mathrm{C}$ & NA & NA & NA & 21 & NA & NA & NA \\
\hline
\end{tabular}

TEMP: temperatura; DA: dia de análise; AMB: ambiente; HI: hipoclorito inorgânico; HO: hipoclorito orgânico; NA: Não Analisado. Letras minúsculas iguais, na mesma coluna, não diferem significativamente no nível de 5\% no teste de Tukey. Letras maiúsculas iguais, na mesma linha, não diferem significativamente no nível de 5\% no teste de Tukey.

suco do que a medição isolada de açúcares ou acidez, sendo, então, uma das formas mais práticas para se estimar a avaliação do sabor (CHITARRA; CHITARRA, 2005).

Os valores de $\mathrm{pH}$ estiveram entre 3,19 e 3,46 durante os 21 dias de armazenamento (Tabela 8). Os frutos armazenados nas temperaturas de $6{ }^{\circ} \mathrm{C}$ e $10{ }^{\circ} \mathrm{C}$ não apresentaram variação significativa nos valores de $\mathrm{pH}$, durante todo o armazenamento. Ocorreu variação significativa nesses valores aos quatro dias de armazenamento, no produto submetido à sanitização com hipoclorito inorgânico, sendo que o produto mantido sob condição ambiente apresentou maiores valores de $\mathrm{pH}$, quando comparado aos demais tratamentos.

$\mathrm{O}$ aumento nos valores de $\mathrm{pH}$ durante o armazenamento de produtos vegetais relaciona-se com a redução nos valores de acidez que normalmente ocorre nos produtos, uma vez que os ácidos presentes nos frutos podem ser utilizados nos processos metabólicos ocorridos no vegetal, na tentativa de manter-se vivo após a colheita. As oscilações nos valores de $\mathrm{pH}$ durante o armazenamento 
Conservação pós-colheita de frutos de Passiflora setacea DC. submetidos a diferentes sanitizantes e temperaturas de armazenamento

Rinaldi, M. M. et al.

Tabela 8. Valores médios de $\mathrm{pH}$ em frutos de Passiflora setacea cv. BRS Pérola do Cerrado, submetidos a diferentes tratamentos.

\begin{tabular}{|c|c|c|c|c|c|c|c|}
\hline \multirow{5}{*}{ TEMP } & \multicolumn{7}{|c|}{ pH } \\
\hline & \multicolumn{3}{|c|}{ DA } & \multirow{4}{*}{ DA } & \multicolumn{3}{|c|}{ TEMP } \\
\hline & \multicolumn{3}{|c|}{ Sanitizante } & & \multicolumn{3}{|c|}{ Sanitizante } \\
\hline & $\mathrm{H}_{2} \mathrm{O}$ & $\mathrm{HI}$ & HO & & $\mathrm{H}_{2} \mathrm{O}$ & $\mathrm{HI}$ & HO \\
\hline & \multicolumn{3}{|c|}{ Dia 0} & & \multicolumn{3}{|c|}{ AMB } \\
\hline AMB & 3,23 aA & 3,23 aA & 3,23 aA & 0 & $3,23 \mathrm{bA}$ & $3,23 \mathrm{bA}$ & $3,23 \mathrm{bA}$ \\
\hline $6^{\circ} \mathrm{C}$ & 3,23 aA & 3,23 aA & 3,23 aA & 4 & $3,27 \mathrm{bB}$ & 3,43 aA & $3,28 \mathrm{abB}$ \\
\hline $10^{\circ} \mathrm{C}$ & $3,23 \mathrm{aA}$ & $3,23 \mathrm{aA}$ & 3,23 aA & 7 & $3,41 \mathrm{aA}$ & $3,37 \mathrm{aAB}$ & 3,33 aB \\
\hline $15^{\circ} \mathrm{C}$ & 3,23 aA & 3,23 aA & 3,23 aA & 14 & NA & NA & NA \\
\hline $20^{\circ} \mathrm{C}$ & 3,23 aA & 3,23 aA & 3,23 aA & 21 & NA & NA & NA \\
\hline \multicolumn{5}{|c|}{ Dia 4} & \multicolumn{3}{|c|}{$6^{\circ} \mathrm{C}$} \\
\hline AMB & $3,28 \mathrm{aB}$ & 3,44 aA & $3,30 \mathrm{aB}$ & 0 & 3,23 aA & $3,23 \mathrm{aA}$ & $3,23 \mathrm{aA}$ \\
\hline $6^{\circ} \mathrm{C}$ & 3,29 aA & $3,31 \mathrm{abA}$ & 3,20 aA & 4 & 3,29 aA & 3,31 aA & $3,20 \mathrm{aA}$ \\
\hline $10^{\circ} \mathrm{C}$ & 3,27 aA & $3,21 \mathrm{bA}$ & $3,22 \mathrm{aA}$ & 7 & 3,27 aA & $3,28 \mathrm{aA}$ & 3,23 aA \\
\hline $15^{\circ} \mathrm{C}$ & $3,30 \mathrm{aA}$ & $3,25 \mathrm{bA}$ & 3,25 aA & 14 & 3,32 aA & 3,39 aA & 3,41 aA \\
\hline $20^{\circ} \mathrm{C}$ & 3,19 aA & $3,26 \mathrm{bA}$ & $3,31 \mathrm{aA}$ & 21 & 3,26 aA & 3,26 aA & 3,27 aA \\
\hline \multicolumn{5}{|c|}{ Dia 7} & \multicolumn{3}{|c|}{$10^{\circ} \mathrm{C}$} \\
\hline AMB & $3,42 \mathrm{bA}$ & $3,38 \mathrm{bB}$ & $3,34 \mathrm{bC}$ & 0 & 3,23 aA & $3,23 \mathrm{aA}$ & $3,23 \mathrm{aA}$ \\
\hline $6^{\circ} \mathrm{C}$ & $3,27 \mathrm{dA}$ & $3,28 \mathrm{cA}$ & $3,23 \mathrm{~dB}$ & 4 & 3,27 aA & 3,21 aA & 3,22 aA \\
\hline $10^{\circ} \mathrm{C}$ & $3,25 \mathrm{~dB}$ & $3,23 \mathrm{~dB}$ & $3,29 \mathrm{cA}$ & 7 & 3,25 aA & $3,23 \mathrm{aA}$ & $3,29 \mathrm{aA}$ \\
\hline $15^{\circ} \mathrm{C}$ & $3,34 \mathrm{cB}$ & $3,26 \mathrm{cC}$ & $3,44 \mathrm{aA}$ & 14 & 3,39 aA & 3,28 aA & 3,42 aA \\
\hline $20^{\circ} \mathrm{C}$ & 3,46 aA & $3,42 \mathrm{aB}$ & $3,43 \mathrm{aB}$ & 21 & 3,25 aA & $3,29 \mathrm{aA}$ & 3,26 aA \\
\hline \multicolumn{5}{|c|}{ Dia 14} & \multicolumn{3}{|c|}{$15^{\circ} \mathrm{C}$} \\
\hline AMB & NA & NA & NA & 0 & $3,23 \mathrm{dA}$ & $3,23 \mathrm{cA}$ & $3,23 \mathrm{dA}$ \\
\hline $6^{\circ} \mathrm{C}$ & $3,32 \mathrm{bc}$ & $3,39 \mathrm{aB}$ & $3,41 \mathrm{abA}$ & 4 & $3,30 \mathrm{cA}$ & $3,25 \mathrm{bB}$ & $3,25 \mathrm{cB}$ \\
\hline $10^{\circ} \mathrm{C}$ & $3,39 \mathrm{aB}$ & $3,28 \mathrm{cC}$ & 3,42 aA & 7 & $3,34 \mathrm{bB}$ & $3,26 b c$ & 3,44 aA \\
\hline $15^{\circ} \mathrm{C}$ & $3,40 \mathrm{aA}$ & $3,30 \mathrm{cB}$ & $3,28 \mathrm{cB}$ & 14 & 3,40 aA & $3,30 \mathrm{aB}$ & $3,28 \mathrm{bB}$ \\
\hline $20^{\circ} \mathrm{C}$ & $3,26 \mathrm{cC}$ & $3,34 \mathrm{bB}$ & $3,39 \mathrm{bA}$ & 21 & NA & NA & NA \\
\hline \multicolumn{5}{|c|}{ Dia 21} & \multicolumn{3}{|c|}{$20^{\circ} \mathrm{C}$} \\
\hline AMB & NA & NA & NA & 0 & $3,23 \mathrm{cA}$ & $3,23 \mathrm{dA}$ & $3,23 \mathrm{dA}$ \\
\hline $6^{\circ} \mathrm{C}$ & 3,26 aA & $3,26 \mathrm{bA}$ & $3,27 \mathrm{aA}$ & 4 & $3,19 \mathrm{dC}$ & $3,26 \mathrm{cB}$ & $3,31 \mathrm{cA}$ \\
\hline $10^{\circ} \mathrm{C}$ & $3,25 \mathrm{aB}$ & 3,29 aA & $3,26 \mathrm{aB}$ & 7 & 3,46 aA & $3,42 \mathrm{aB}$ & $3,43 \mathrm{aB}$ \\
\hline $15^{\circ} \mathrm{C}$ & NA & NA & NA & 14 & $3,26 \mathrm{bc}$ & $3,34 \mathrm{bB}$ & $3,39 \mathrm{bA}$ \\
\hline $20^{\circ} \mathrm{C}$ & NA & NA & NA & 21 & NA & NA & NA \\
\hline
\end{tabular}

TEMP: temperatura; DA: dia de análise; AMB: ambiente; HI: hipoclorito inorgânico; HO: hipoclorito orgânico; NA: Não Analisado. Letras minúsculas iguais, na mesma coluna, não diferem significativamente no nível de $5 \%$ no teste de Tukey. Letras maiúsculas iguais, na mesma linha, não diferem significativamente no nível de 5\% no teste de Tukey.

geralmente correspondem às características intrínsecas da amostra, à desuniformidade no ponto de colheita dos frutos e a outros fatores.

\section{Conclusões}

A melhor condição de armazenamento para os frutos de Passiflora setácea DC. é sob refrigeração na temperatura de $10{ }^{\circ} \mathrm{C}$ e $90 \%$ de umidade relativa. Na temperatura de $6{ }^{\circ} \mathrm{C}$, os frutos apresentaram chilling injury, não sendo recomendado o armazenamento dos mesmos nesta condição. A utilização dos sanitizantes não aumentou a vida útil dos frutos, não sendo assim recomendados para utilização nos frutos de Passiflora setácea DC. .

A vida útil pós-colheita dos frutos foi inferior a quatro dias de armazenamento, mesmo sob refrigeração; observou-se que, assim como o maracujazeiro azedo comercial, frutos de $P$. setacea perdem massa fresca e apresentam o enrugamento da casca após quatro dias de armazenamento. No entanto, de acordo com os valores de $\mathrm{pH}$, acidez titulável e sólidos solúveis, essas características não inviabilizam o consumo da polpa após sete dias de armazenamento.

\section{Agradecimentos}

Ao Conselho Nacional de Desenvolvimento Científico e Tecnológico (CNPq) Projeto N 404847/2012-09 pelo apoio financeiro, e à Empresa Brasileira de Pesquisa Agropecuária (Embrapa) pelo apoio no desenvolvimento da pesquisa. 
Conservação pós-colheita de frutos de Passiflora setacea DC. submetidos a diferentes sanitizantes e temperaturas de armazenamento

Rinaldi, M. M. et al.

\section{Referências}

AMARO, A. P.; MONTEIRO, M. Rendimento de extração da polpa e características físico químicas do maracujá amarelo (Passiflora edulis f. flavicarpaSims. Deg.) produzido por cultivo orgânico e convencional em relação à cor da casca. Alimentos e Nutrição, v. 1, n. 1, p. 171-184, 2001.

ARRUDA, M. C.; FISCHER, I. H.; JERONIMO, E. M.; ZANETTE, M. M.; DA SILVA, B. L. Efeito de produtos químicos e temperaturas de armazenamento na pós-colheita de maracujá-amarelo. Semina: Ciências Agrárias, v. 32, n. 1, p. 201-208, 2011.

ATAÍDE, E. M.; OLIVEIRA, J. C.; RUGGIERO, C. Florescimento e frutificação do maracujazeiro silvestre Passiflora setacea D. C. cultivado em Jaboticabal, SP. Revista Brasileira de Fruticultura, v. 34, n. 2, p. 377-381, 2012.

BRASIL. Ministério da Agricultura e do Abastecimento. Instrução Normativa $n^{\circ}$ 01, de 7 de janeiro de 2000. Anexo VII - Regulamento técnico para fixação dos padrões de identidade e qualidade para polpa de maracujá. Diário Oficial [da] República Federativa do Brasil, Brasília, DF, 10 jan. 2000.

BRUCKNER, C. H.; MELETTI, L. M. M.; OTONI, W. C.; ZERBINI JÚNIOR, F. M. Maracujazeiro. In: BRUCKNER, C. H. (Ed.). Melhoramento de fruteiras tropicais. Viçosa: Editora UFV, 2002. p. 373-409.

CARVALHO, C. R. L.; MANTOVANI, D. M. B.; CARVALHO, P. R. N.; MORAES, R. M. M. Análises químicas de alimentos. Campinas: ITAL, 1990. $121 \mathrm{p}$.

CERQUEIRA-SILVA, C.; CONCEIÇÃO, L.; SOUZA, A.; CORRÊA, $R$. A history of passion fruit woodiness disease with emphasis on the current situation in Brazil and prospects for Brazilian passion fruit cultivation. European Journal of Plant Pathology, v. 139, n. 2, p. 255-264, 2014.

CHITARRA, M. I. F.; CHITARRA, A. B. Pós-colheita de frutas e hortaliças: fisiologia e manuseio. Lavras: ESAL; FAEPE, 2005. $785 \mathrm{p}$.

DURIGAN, J. F. Colheita e conservação pós-colheita. In: SIMPÓSIO BRASILEIRO SOBRE A CULTURA DO MARACUJAZEIRO, 5., 1998, Jaboticabal. Anais... Jaboticabal: FUNEP, 1998. 388 p.

EMPRESA BRASILEIRA DE PESQUISA AGROPECUÁRIA EMBRAPA. Lançamento da cultivar de maracujazeiro silvestre BRS Pérola do Cerrado. Planaltina: Embrapa Cerrados, 2015. Disponível em: <http://www.cpac.embrapa.br/lancamentoperola/>. Acesso em: 10 fev. 2015

FALEIRO, F. G.; JUNQUEIRA, N. T. V.; OLIVEIRA, E. J.; MACHADO, C. F.; PEIXOTO, J. R.; COSTA, A. M.; GUIMARÃES, T. G.; JUNQUEIRA, K. P. Caracterização de germoplasma e melhoramento genético do maracujazeiro assistidos por marcadores moleculares Fase II: resultados de pesquisa 2008-2012. Planaltina: Embrapa Cerrados, 2014. 102 p. (Documentos, 324).
FEDERAÇÃO DA AGRICULTURA DO ESTADO DO PARANÁ - FAEP. Classificação do maracujá-amarelo. Curitiba, 2015. Disponível em: <http://www.faep.com.br/comissoes/frutas/cartilhas/frutas/ maracuja.htm>. Acesso em: 10 jul. 2015.

FORTALEZA, J. M.; PEIXOTO, J. R.; JUNQUEIRA, N. J. V.; OLIVEIRA, A. T.; RANGEL, L. E. P. Características físicas e químicas em nove genótipos de maracujá-azedo cultivado sob três níveis de adubação potássica. Revista Brasileira de Fruticultura, v. 27 , n. 1, p. 124-127, 2005.

GOMES, T. S.; CHIBA, H. T.; SIMIONATO, E. M. R. S.; SAMPAIO, A. C. Monitoramento da qualidade da polpa de maracujá-amareloseleção AFRUVEC, em função do tempo de armazenamento do frutos. Revista Alimentos e Nutrição, v. 17, n. 4, p. 401-405, 2006.

HUNTER ASSOCIATES LABORATORY - HUNTERLAB. Insight on color: CIE $L^{*} a^{*} b^{*}$ color scale. Reston, 2008.

INSTITUTO FNP. AGRIANUAL - Anuário da Agricultura Brasileira São Paulo, 2016. 472 p.

MACHADO, S. S.; CARDOSO, R. L.; MATSUURA, F. C. A. U.; FOLEGATTI, M. I. S. Caracterização física e físico-química de frutos de maracujá amarelo provenientes da região de Jaguaquara - Bahia. Magistra, v. 15, n. 2, p. 229-233, 2003.

PITA, J. S. L. Caracterização físico-química e nutricional da polpa e farinha da casca de maracujazeiro do mato e amarelo. 2012. 80 f. Dissertação (Mestrado em Engenharia de Alimentos)Universidade Estadual do Sudoeste da Bahia, Itapetinga, 2012.

RAIMUNDO, K.; MAGRI, R. S.; SIMIONATO, E. M. R. S.; SAMPAIO, A. C. Avaliação física e química da polpa de maracujá congelada comercializada na região de Bauru. Revista Brasileira de Fruticultura, v. 31, n. 4, p. 539-543, 2009.

ROTILI, M. C. C.; VORPAGEL, J. A.; BRAGA, G. C.; KUHN, O. J.; SALIBE, A. B. Atividade antioxidante, composição química e conservação de maracujá-amarelo embalado com filme de PVC. Revista Brasileira de Fruticultura, v. 35, n. 4, p. 942-952, 2013.

SILVA, F. A. S. Software ASSISTAT. Versão 7.7 Beta. Rio de Janeiro: INPI, 2015. Disponível em: <http://www.assistat.com>. Acesso em: 3 maio 2015

SILVA, L. J. B.; SOUZA, M. L.; ARAUJO NETO, E.; MORAIS, A. P. Revestimentos alternativos na conservação pós-colheita de maracujá-amarelo. Revista Brasileira de Fruticultura, v. 31, n. 4, p. 995-1003, 2009.

TAVARES, J. T. Q.; SILVA, C. L. A.; CARVALHO, L. A.; SILVA, M. A.; SANTOS, C. M. G.; TEIXEIRA, L. J.; SANTANA, R. S. Aplicação pós - colheita de cloreto de cálcio em maracujá amarelo. Magistra, v. 15, n. 1, p. 7-12, 2003.

VENÂNCIO, J. B.; SILVEIRA, M. V.; FEHLAUER, T. V.; PEGORARE, A. B.; RODRIGUES, E. T.; ARAÚJO, W. F. Tratamento hidrotérmico e cloreto de cálcio na pós-colheita de maracujá-amarelo. Científica, v. 41, n. 2, p. 122-129, 2013. 